\title{
Design of Positive, Negative, and Alternating Sign Generalized Logistic Maps
}

\author{
Wafaa S. Sayed, ${ }^{1}$ Ahmed G. Radwan, ${ }^{1,2}$ and Hossam A. H. Fahmy ${ }^{3}$ \\ ${ }^{1}$ Engineering Mathematics and Physics Department, Faculty of Engineering, Cairo University, Giza 12613, Egypt \\ ${ }^{2}$ Nanoelectronics Integrated Systems Center, Nile University, Cairo 12588, Egypt \\ ${ }^{3}$ Electronics and Electrical Communications Department, Faculty of Engineering, Cairo University, Giza 12613, Egypt
}

Correspondence should be addressed to Ahmed G. Radwan; agradwan@ieee.org

Received 1 April 2015; Accepted 2 June 2015

Academic Editor: Agustin Martin

Copyright (C) 2015 Wafaa S. Sayed et al. This is an open access article distributed under the Creative Commons Attribution License, which permits unrestricted use, distribution, and reproduction in any medium, provided the original work is properly cited.

\begin{abstract}
The discrete logistic map is one of the most famous discrete chaotic maps which has widely spread applications. This paper investigates a set of four generalized logistic maps where the conventional map is a special case. The proposed maps have extra degrees of freedom which provide different chaotic characteristics and increase the design flexibility required for many applications such as quantitative financial modeling. Based on the maximum chaotic range of the output, the proposed maps can be classified as positive logistic map, mostly positive logistic map, negative logistic map, and mostly negative logistic map. Mathematical analysis for each generalized map includes bifurcation diagrams relative to all parameters, effective range of parameters, first bifurcation point, and the maximum Lyapunov exponent (MLE). Independent, vertical, and horizontal scales of the bifurcation diagram are discussed for each generalized map as well as a new bifurcation diagram related to one of the added parameters. A systematic procedure to design two-constraint logistic map is discussed and validated through four different examples.
\end{abstract}

\section{Introduction}

Chaotic iterated maps are essential in both modeling and information processing in many fields. This explains the need for their hardware analog and digital realizations, for example, [1-10]. The conventional logistic map is a famous iterative map based on first order nonlinear difference equation which can model growth rate and is given by

$$
x_{n+1}=\lambda x_{n}\left(1-x_{n}\right) ; \quad \lambda \in R^{+},
$$

where $\lambda$ is the population growth rate or fertility coefficient and $x_{n} \in[0,1]$ is the relative population size at a discrete time instant $n$.

The map was initially popularized in a paper by the biologist May [11] as a discrete time demographic model, analogous to the logistic equation first created by Verhulst [12]. A detailed mathematical analysis of the map and its properties has also been presented in [13]. Specifically, the applications of the logistic map have increased during the last few decades, for example, in fields as biology [14], chemistry [15], physics [16], secure data and image transfer [17, 18], random number generation for chaos based communication [19-23], circuit applications [24], traffic [25], financial modeling, and business cycle theory [26-28].

The bifurcation diagram shown in Figure 1 is a basic characteristic of the logistic map which represents the sudden appearance of qualitatively different solutions as $\lambda$ is varied. For $\lambda<3$, the system has one stable fixed point. For $3<\lambda<$ $1+\sqrt{6}$, the output oscillates between two fixed points; that is, the recurrence converges to a period-2 orbit. This period's doubling, quadrupling, and so forth, are called bifurcation which accompanies the onset of chaos. Starting at $\lambda=1+\sqrt{8}$, the recurrence converges to a period- 3 orbit which implies the presence of chaos according to Sharkovskii's theorem [29]. Consequently, the output continues bifurcation until $\lambda$ reaches the value 4 which shows chaos in the whole range as shown in Figure 1. A chaotic system exhibits infinite number of periodic orbits, some of which could be of arbitrarily long period.

Conventional chaotic systems, especially discrete 1D maps, are highly rich in information and indications that constitute a large portion of the basic study of chaos theory. 


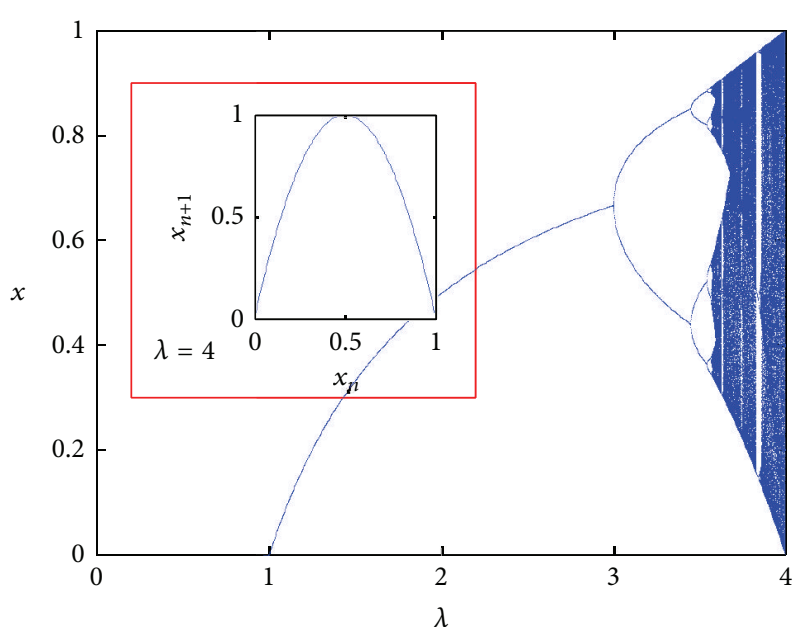

FIGURE 1: Bifurcation diagram and graph of the conventional logistic map.

However, modeling dynamics in natural phenomena and stochastic processes, as well as demand on random number generation for multiple purposes, requires novel chaotic systems. Consequently, generalized maps have been proposed in previous researches [30-33]. Yet, they mostly depend on rather complicated equations that might even be considered 2D maps. Moreover, the parameters of generalized chaotic function proposed in [30] have been adapted to fit specific encryption algorithm. Thus, the map has not been mathematically analyzed in a generic way. Other generalized logistic, tent, and sine maps have been recently investigated in [34-36]. In this paper, generalizations providing extra degrees of freedom are proposed that could be adapted to fit certain specifications, keeping the advantage of simple mathematical relation using $1 \mathrm{D}$ maps only. Requirements on the key points of the resulting bifurcation diagram or the range of the system output could be achieved directly and simply through controlling the values of the parameters which are governed by simple relations. The simplest of which does not require any modifications from the viewpoint of implementation other than using a signed register for both the system parameter $\lambda$ and the output $x$. However, for a legible comprehensive analysis, we define the sign of the parameter explicitly confining its value to the set of positive reals $R^{+}$. Moreover, further generalizations are proposed that allow scaling of the bifurcation diagrams.

The rest of the paper is organized as follows. Section 2 lists the possible variations on the relation representing the map, according to the signs of different control parameters and the resulting output ranges. The generalized form of these variations is given by

$$
x_{n+1}= \pm \lambda x_{n}\left(a \pm b x_{n}\right) ; \quad \lambda, a, b \in R^{+} .
$$

Based on the maximum chaotic range of the output, the proposed maps can be classified as positive logistic map, mostly positive logistic map, negative logistic map, and mostly negative logistic map. These maps differ in the ranges of both $\lambda$ and $x$, the symmetry, the key points of the bifurcation diagram, and the possible applications that could employ the resulting ranges. Section 3 analyzes the generalized logistic maps and studies how to design any of them to suit certain specifications, proposing three scaling cases. The parameters $(a, b)$ may take one of three cases: $(a, b), a, b \in R^{+}$called the independent scaling case, $(1, b)$ called the vertical scaling case, and $(a, 1)$ called the zooming case. The choice of these names depends on the effect of the added parameter(s) on the bifurcation diagram. The three proposed parameterized versions are analyzed for each map from the viewpoint of iteration effect, ranges of $\lambda$ and $x$, the fixed points, the bifurcation diagrams, and the maximum Lyapunov exponent with respect to all system parameters. A new bifurcation diagram versus the parameter $a$ is introduced. Section 4 summarizes the results and provides the general schematic of the bifurcation diagram versus $\lambda$ as a function of the other system parameters, as well as the general schematic of the newly proposed bifurcation diagram versus $a$. Four different design examples are presented in Section 5 to verify the provided design procedure according to the general schematic. The designed maps are validated for usage in encryption applications through a simple text encryption scheme. Finally, the last section concludes the contributions of the paper.

\section{Generalizations of the Logistic Map and Their Applications}

Properties of the conventional discrete 1D logistic map and its applications were extensively discussed in the literature. Recent models of stochastic behaviors represent a great challenge and strong motivation to devise methods for designing generalized maps. Requirements on the key points of the resulting bifurcation diagram or the range of the system output could be achieved in many ways. In this section, we propose a simple and efficient way through controlling the values of multiple parameters governed by simple relations. The proposed maps and their usefulness to real world applications are discussed.

2.1. The Proposed Maps. We define the following parameterized maps controlled by the set of parameters $(a, b, \lambda)$ such that $a, b, \lambda \in R^{+}$. The names of the maps have been chosen carefully to represent the maximum chaotic range of the output.

Definition 1. Variations on the sign of the generalized logistic map represented by (2) can be subdivided into two types according to the maximum chaotic range of the output:

(1) Single sign maps:

(a) Positive logistic map given by

$$
f_{1}\left(x_{n}\right)=x_{n+1}=\lambda x_{n}\left(a-b x_{n}\right) .
$$

(b) Negative logistic map given by

$$
f_{3}\left(x_{n}\right)=x_{n+1}=\lambda x_{n}\left(a+b x_{n}\right),
$$

where $f_{3}(x)=-f_{1}(-x)$. The bifurcation diagram of this map is the reflected image of $f_{1}(x)$ 


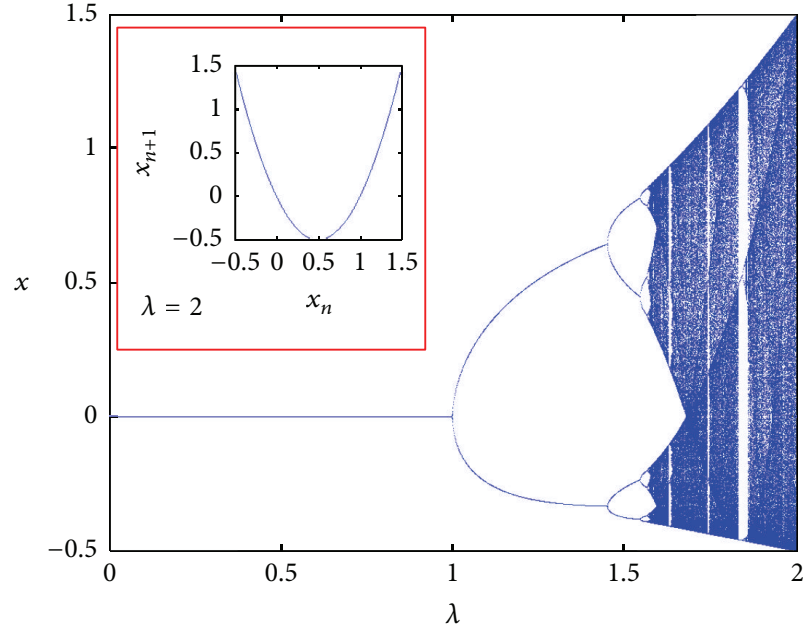

(a) $f_{2}(x)=-\lambda x(1-x)$

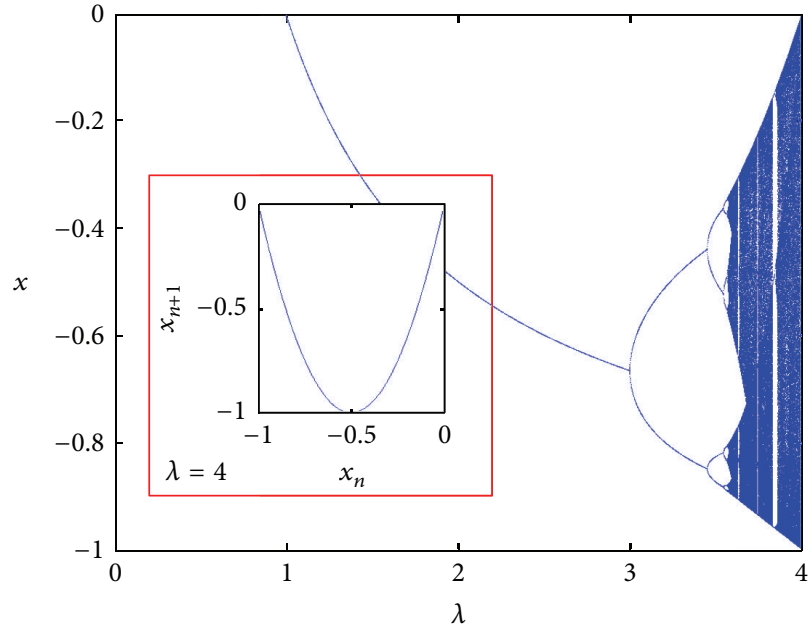

(b) $f_{3}(x)=\lambda x(1+x)$

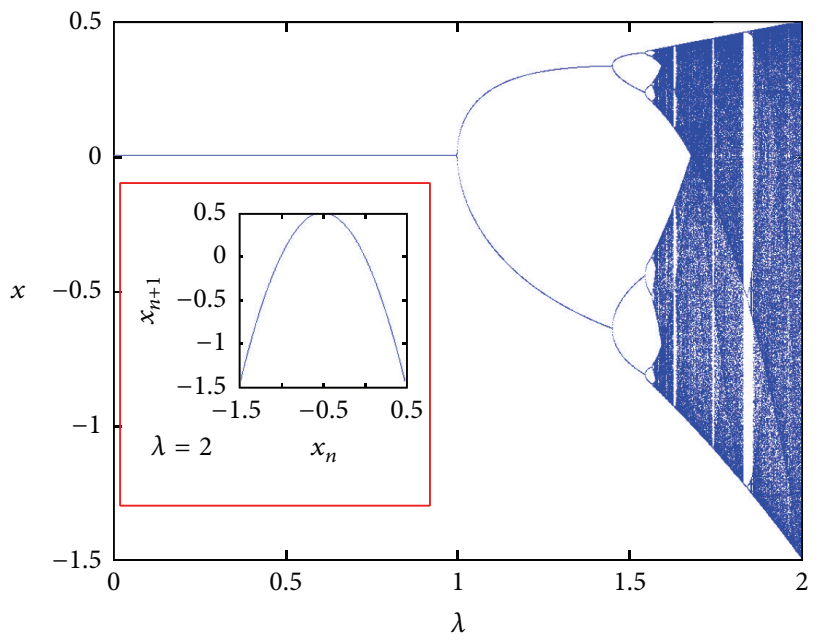

(c) $f_{4}(x)=-\lambda x(1+x)$

FIGURE 2: Bifurcation diagrams and graphs for the proposed unity scaling maps.

about the horizontal axis $(\lambda)$, yielding exactly the same range of output magnitude, but with opposite sign.

(2) Alternating sign maps:

(a) Mostly positive logistic map given by

$$
f_{2}\left(x_{n}\right)=x_{n+1}=-\lambda x_{n}\left(a-b x_{n}\right) .
$$

(b) Mostly negative logistic map given by

$$
f_{4}\left(x_{n}\right)=x_{n+1}=-\lambda x_{n}\left(a+b x_{n}\right),
$$

where $f_{4}(x)=-f_{2}(-x)$. Thus, the relation between the two maps is similar to that between $f_{1}$ and $f_{3}$.

Figure 2 shows the bifurcation diagrams for three maps in the case of unity scaling $(a=b=1)$, and the graphs of their equations at maximum $\lambda$. The properties of $f_{1}$ have been previously shown in Figure 1. Table 1 illustrates the main differences between the proposed maps in the case of unity scaling. The output ranges of the proposed maps allow more flexibility in chaotic modeling of finance [26, 27], traffic, weather forecasting $[37,38]$, and many other fields. The wider output range and the asymmetry recognized in the resulting bifurcation diagrams of these maps could also be added to their advantages as they imply more unpredictability, in addition to the earlier onset of chaos in both of the alternating sign maps compared to the other two maps. The rest of the paper provides a detailed analysis of three different parameterized versions for each of the proposed maps. But first, we devote the rest of this section to present a quick, but not exhaustive, review of related real-world applications, followed by a study of the main properties of the negative and alternating sign maps which have not been covered before in the literature to the best of our knowledge. Various experiments have shown that some phenomena exhibit dynamical behaviors which could only be modeled by generalized maps with modulated parameters as conventional maps are not sufficient to 
TABLE 1: Main differences between the proposed unity scaling maps.

\begin{tabular}{lcc}
\hline Map & Range of $\lambda$ & Range of $x$ \\
\hline$\lambda x(1-x)$ & {$[0,4]$} & {$[0,1]$} \\
$-\lambda x(1-x)$ & {$[0,2]$} & {$[-0.5,1.5]$} \\
$\lambda x(1+x)$ & {$[0,4]$} & {$[-1,0]$} \\
$-\lambda x(1+x)$ & {$[0,2]$} & {$[-1.5,0.5]$} \\
\hline
\end{tabular}

model $[39,40]$. On the other hand, proposed generalizations on existing maps enhance their unpredictability and increase their reliability in secure communication and encryption. We believe that there is still a gap which needs to be filled between areas of research in which some biologists, physicists, and economists propose new models and the field of nonlinear dynamics and chaos in which researchers could come up with chaotic systems suitable for predicting the behavior of these models. Examples of models that could employ the proposed maps are discussed below.

2.2. Negative Probabilities and Probabilities above Unity. Axioms of Kolmogorov's probability theory [41] have the rule $0 \leq P(A) \leq 1$ since $P(A)$ describes the probability of occurrence of event $A$ or the probability of the outcome of an experiment which must be a positive real number. However, these axioms do not provide the complete picture of stochastic reality. Possibilities to extend the probability theory to describe numerous physical models with negative probabilities and even probabilities more than one have been mathematically discussed, as in [42], and have been used in solving several problems [43-45] and paradoxes, for example, Einstein-Podolsky-Rosen paradox [46]. These out of the expected range probabilities are allowed by quasiprobability distributions that may apply to unobservable events or conditional probabilities. The idea of negative probabilities first arised in physics and particularly in quantum mechanics based on ideas of Wigner [47], Dirac [48], and Feynman [49]. Later on, many physicists and scientists [50-53] have argued for the necessity of extended probabilities in quantum theories and that their appearance is one of the main differences between classical and quantum theories. For instance, the probabilities of existence of positive and negative-energy photons or the emissions and absorptions of photons equal 2 and -2 , respectively [54].

Negative probabilities have more recently been applied to mathematical finance. In quantitative finance, most probabilities are not real probabilities but theoretical ones called pseudoprobabilities. The concept of risk-neutral or pseudoprobabilities is a popular concept in finance which has been numerously applied as in $[55,56]$. It has been shown how negative probabilities can be applied to financial option pricing. For example, calculating the probability of a price going up or down could be simplified by allowing such pseudoprobabilities to be negative in certain cases as pointed out by Haug [57]. A generalized version of the well-known Cox, Ross, and Rubinstein binomial tree (CRR tree) [58] has been considered. The tree is often used to price a variety of derivatives instruments where different probabilities could be yielded which might lie outside the interval $[0,1]$. For example, one can get a risk-neutral up probability of 1.3689 and a down probability of -0.3689 . A rigorous mathematical definition of negative probabilities in finance and their properties has been recently derived by Burgin and Meissner [59]. The authors provide several situations in which negative probabilities occurred in finance as well as negative interest rates: overgenerated electricity in Norway that resulted in its price going negative a few hours during the night, the lender paying the bank interest rate in addition to the money in the 1970s in Switzerland, "repos," that is, repurchase agreements traded at negative interest rates which took place in Japan and USA in 2003, and negative nominal interest rates that occurred in the worldwide 2008/2009 financial crisis.

2.3. Alternating Sign Logistic Maps. A study of the properties of the logistic map with negative control parameter $r \in[-2,0]$ that is quite similar to our mostly positive logistic map has been conducted in [60]. Consider the recurrence $x_{n+1}=$ $-\lambda x_{n}\left(1-x_{n}\right)$, graphically represented by the parabola $f(x)=$ $-\lambda x(1-x)$; the critical point of the parabola is $x_{c}$ such that

$$
\begin{aligned}
f^{\prime}\left(x_{c}\right) & =0 \longrightarrow x_{c}=0.5 \\
f\left(x_{c}\right) & =f_{\min }=-\frac{\lambda}{4}
\end{aligned}
$$

For stable steady state response, all the outputs of the recurrence relation should be limited within a closed set. This closure property could be guaranteed by setting a maximum value for $\lambda$. From the symmetry of the curve, this value can be obtained by forcing

$$
\left|f_{\min }\right|=x_{c} \longrightarrow \frac{\lambda_{\max }}{4}=0.5 \longrightarrow \lambda_{\max }=2 .
$$

Figure 3 shows the graph of the equation for different values of $\lambda=1.6,2$, and 2.5, where the map exhibits bounded output if and only if $\lambda \in[0,2]$ as expected before. For $\lambda=2$, the values of $x$ at $u_{1}$ and $u_{2}$ are -0.5 and 1.5, respectively; that is, closed set responses are yielded as $x \in[-0.5,1.5]$. Figure 3 also shows the values of $x$ carrying out 10,000 iterations of the recurrence. The time waveforms indicate the stability of the recurrence for $0 \leq \lambda \leq 2$ as it exhibits a bounded solution. On the other hand, the range $\lambda>2$ shows complete instability of the solution as it diverges to $+\infty$ starting the fifteenth iteration. This illustrative discussion can be rather expressed more formally as follows.

Definition 2. Consider the map given by $f(x)=-\lambda x(1-x)$, the point $u_{1}$ is the one whose coordinates equal the map minimum value $f_{\min }$, and $u_{2}$ is the nontrivial fixed point or the intersection of the curves $y=f(x)$ and $y=x$. The map is stable if and only if the image of $u_{1}\left(f\left(u_{1}\right)\right)$ is less than or equal to $u_{2}$, such that $x \in\left[u_{1}, u_{2}\right]$. This is achieved as $\lambda \in[0,2]$.

The previous method for calculation and verification of the range of values $\lambda$ and $x$ that guarantee bounded responses has not been conducted in [60]. Instead, the value $\lambda_{\max }=2$ and the interval $x \in[-0.5,1.5]$ were used directly in addition to a computer aided plot of the bifurcation diagram that ensures those values. However, our previous method requires 

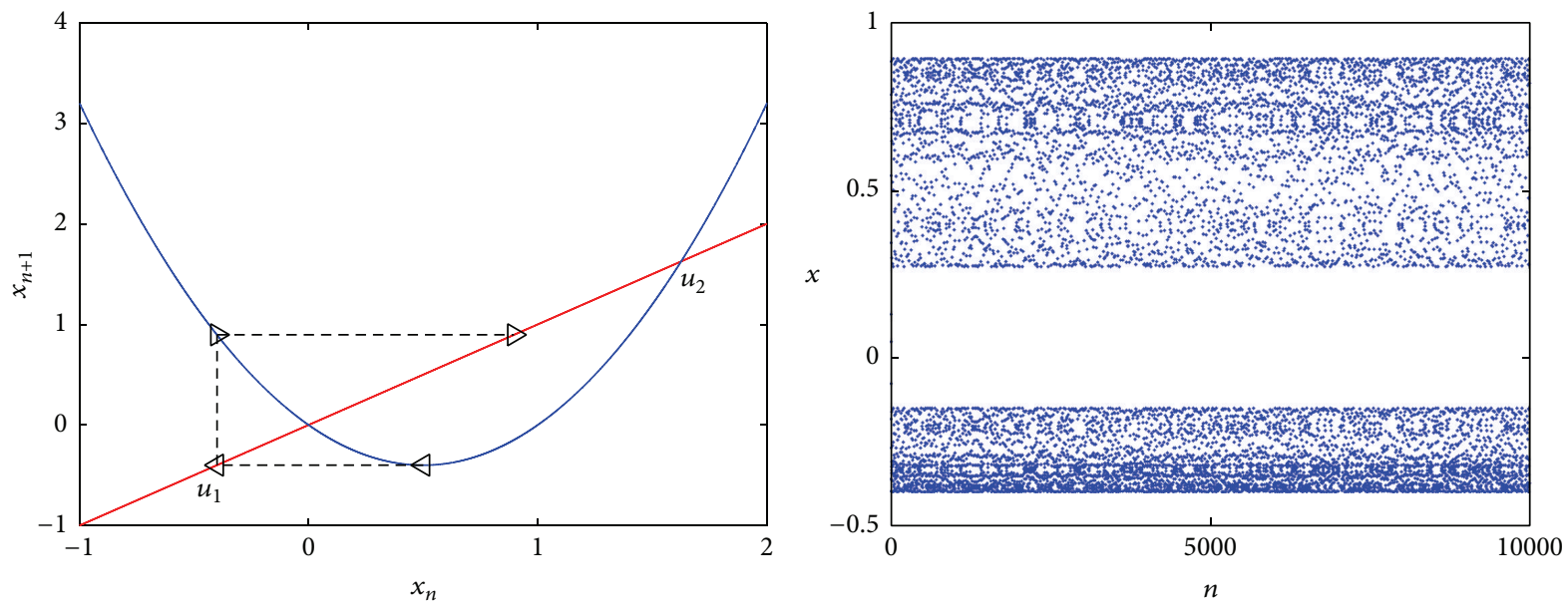

(a)
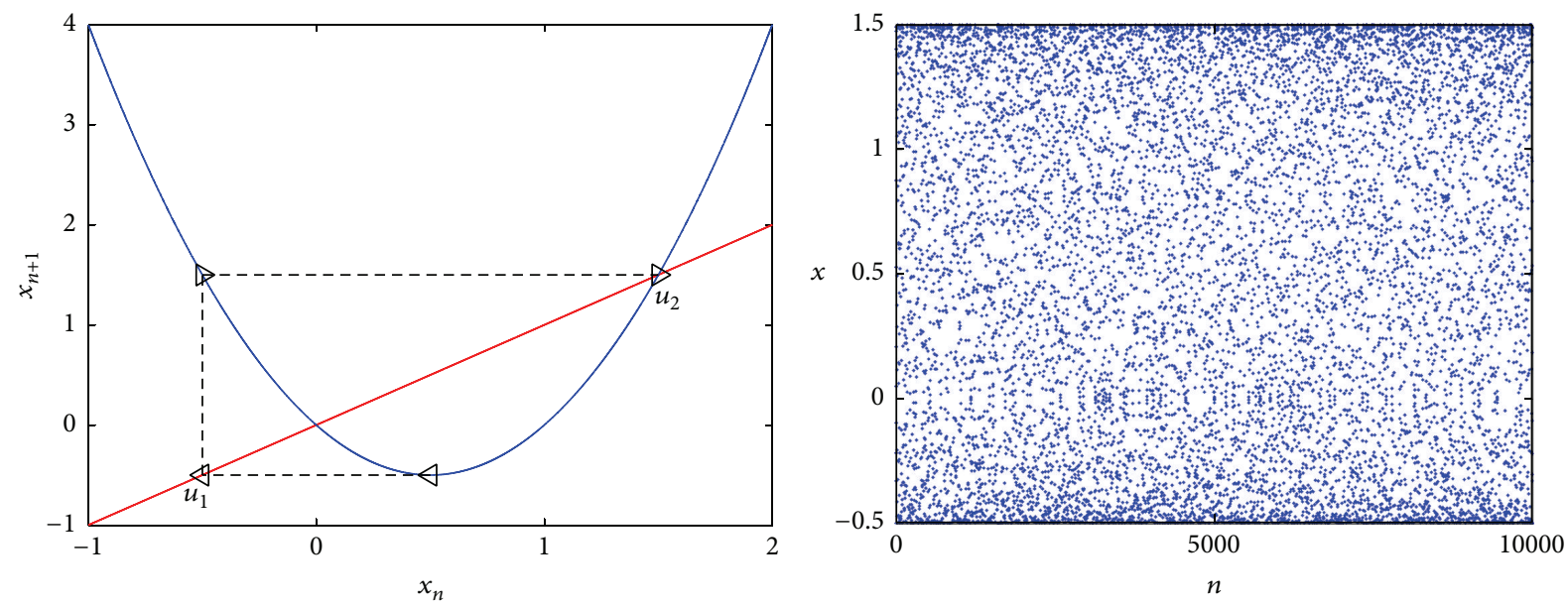

(b)
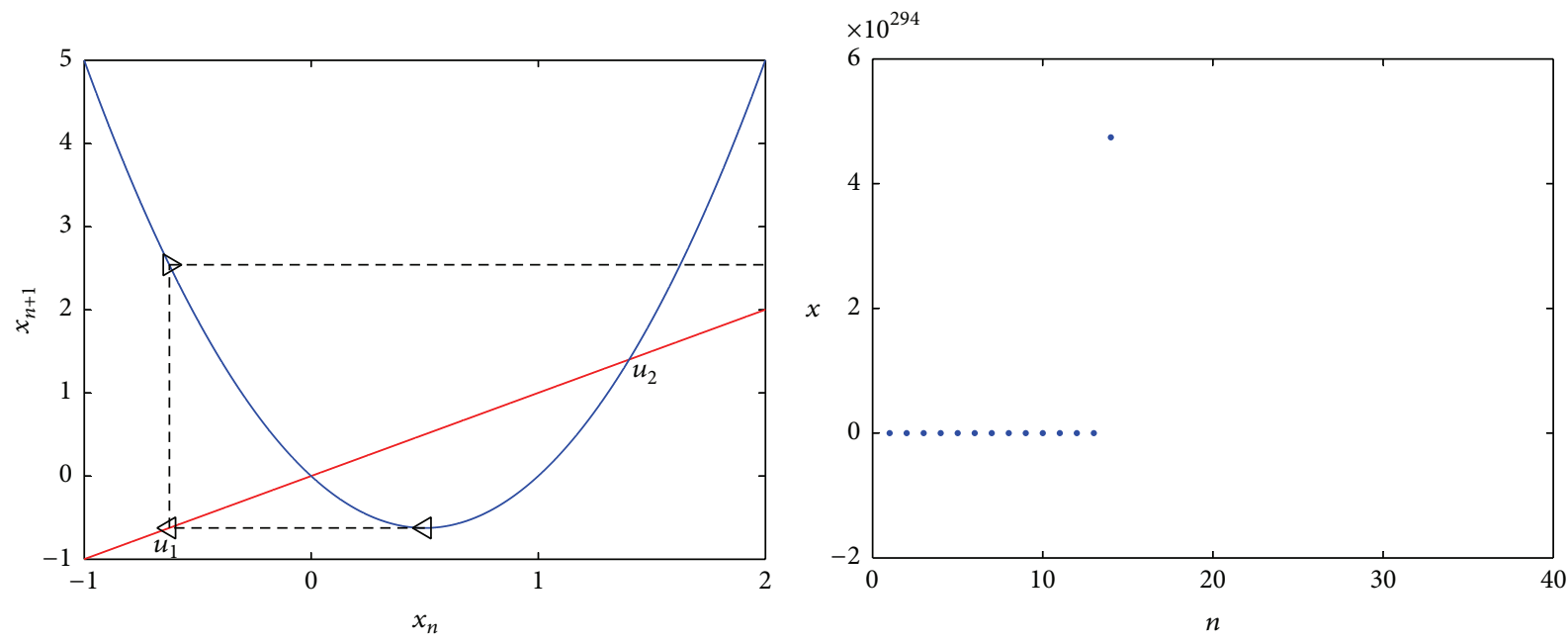

(c)

FIGURE 3: Maximum value of $\lambda$ for mostly positive logistic map: (a) $\lambda=1.6$, (b) $\lambda=2$, and (c) $\lambda=2.5$. 
iterating several values of $\lambda$ and/or an initial assumption. That is why we propose a more generic procedure of specifying the parameters' ranges that could be detailed as follows with the aid of Figure 4 where a map is defined as a function whose domain (input) space and range (output) space are the same [29].

(1) The lower bound on the range of the map is $f_{\min }=$ $f\left(x_{c}\right)$ which is the same as the lower bound on the domain $x_{\min }$. From (7),

$$
f\left(x_{c}\right)=-\frac{\lambda_{\max }}{4} .
$$

(2) The upper bound on the range is $f\left(x_{\min }\right)$, that is, $f\left(f\left(x_{c}\right)\right)$. So, the upper bound is equal to

$$
f\left(f\left(x_{c}\right)\right)=-\lambda_{\max }\left(-\frac{\lambda_{\max }}{4}\right)\left(1+\frac{\lambda_{\max }}{4}\right)
$$

which is a function of $\lambda_{\max }$.

(3) Then, we equate this value to the map equation to get the corresponding solutions for the values of $x: x_{\min }$ and $x_{\max }$, respectively:

$$
\frac{\lambda_{\max }^{2}}{4}+\frac{\lambda_{\max }^{3}}{16}=-\lambda_{\max } x(1-x) .
$$

Rename the left hand side of the previous equation to $\lambda_{\max } C$; it reduces to

$$
C=-x+x^{2}
$$

Thus,

$$
\begin{aligned}
& x_{\min }=-\frac{\lambda_{\max }}{4}, \\
& x_{\max }=1+\frac{\lambda_{\max }}{4} .
\end{aligned}
$$

(4) The domain of the map is $D=\left[x_{\min }, x_{\max }\right]$, while its range is $R=\left[f\left(x_{c}\right), f\left(f\left(x_{c}\right)\right)\right]$. We equate the lower and upper bounds of both intervals, respectively, to get $\lambda_{\text {max }}$ :

$$
1+\frac{\lambda_{\max }}{4}=\frac{\lambda_{\max }^{2}}{4}+\frac{\lambda_{\max }^{3}}{16} .
$$

This equation could be solved to get three values among which one value is positive which is $\lambda_{\max }=2$.

(5) Hence, we get $x_{\min }$ and $x_{\max }$ by substitution for $\lambda_{\max }$ as follows:

$$
x_{\min }=-\frac{1}{2},
$$

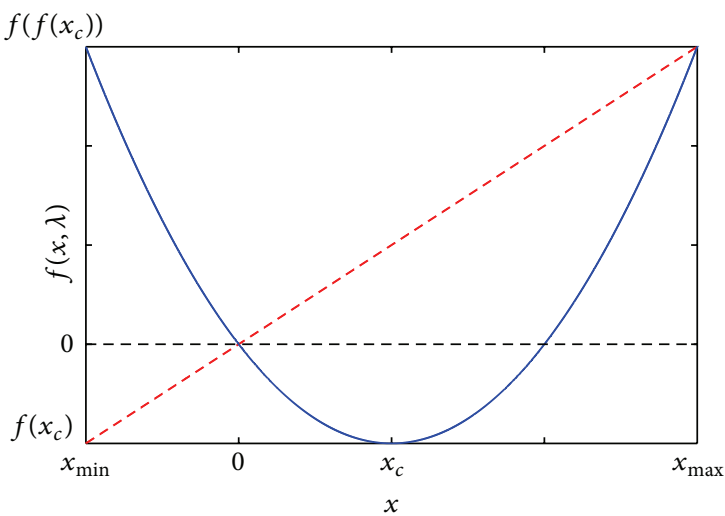

Figure 4: Domain and range of $f(x)=-\lambda_{\max } x(1-x)$.

$$
x_{\max }=\frac{3}{2} .
$$

The mathematical analysis of the fixed and periodic points of mostly positive logistic map in the case of unity scaling is quite similar to that of the conventional logistic map. For $1<\lambda<\sqrt{6}-1$, the recurrence converges to a period-2 orbit, followed by a sequence of periodic doubling. Starting at $\lambda=\sqrt{8}-1$, the recurrence converges to a period-3 orbit. The output continues bifurcation until $\lambda$ reaches the value 2 which exhibits the maximum chaotic behavior. In addition to the bifurcation diagram and the graph of the equation shown in Figure 2(a), the time waveforms starting at different initial points $x_{0}=0.05$ and $x_{0}=0.06$ are shown in Figure 5. These responses demonstrate the maximum chaotic behavior of the map at $\lambda=2$. Figure 5(c) shows the difference between these two responses; the large number of nonzero points indicates the sensitive dependence on initial conditions which is a main property of chaotic systems.

The other two proposed maps: negative logistic map and mostly negative logistic map, are related to positive and mostly positive logistic maps, respectively, as previously mentioned. Thus, for parameterized maps, we analyze both positive and mostly positive logistic maps because the results for the other two maps could be deduced similarly.

\section{Analysis and Results of the Parameterized Maps}

For each map of those presented in the previous section, the resulting bifurcation diagram can be designed with the desired key points using $(a, b)$ as scaling parameters. The parameterized cases studied are $(a, b)$, such that $a, b \in$ $R^{+},(1, b)$, and $(a, 1)$. The following subsections provide the analysis and results of these parameterized cases.

3.1. Independent Scaling $x_{n+1}= \pm \lambda x_{n}\left(a \pm b x_{n}\right)$. In this map, two parameters $a$ and $b$ are added to be capable of scaling both the horizontal and the vertical axes of the bifurcation diagram independently. The analysis presented in this subsection is used later on in Section 5 to design any logistic map with specific characteristics. 


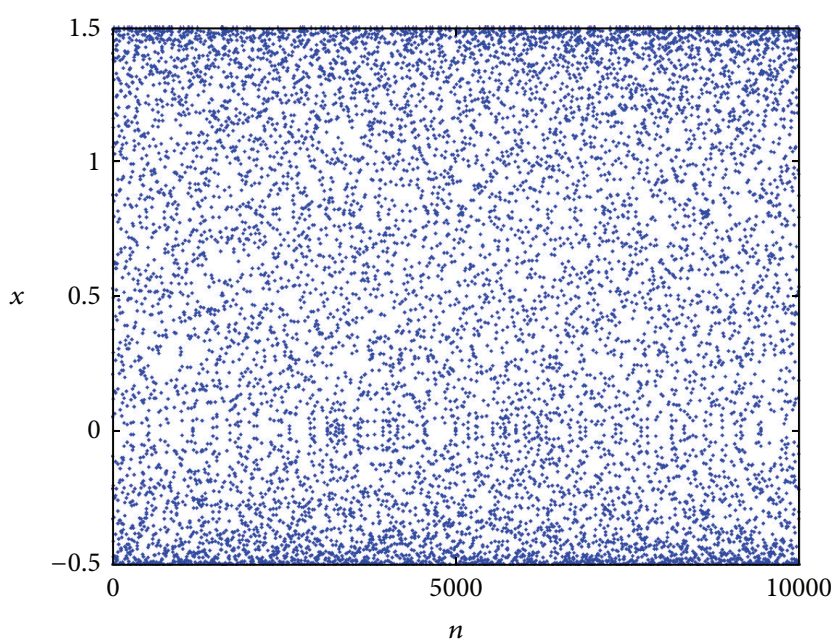

(a)

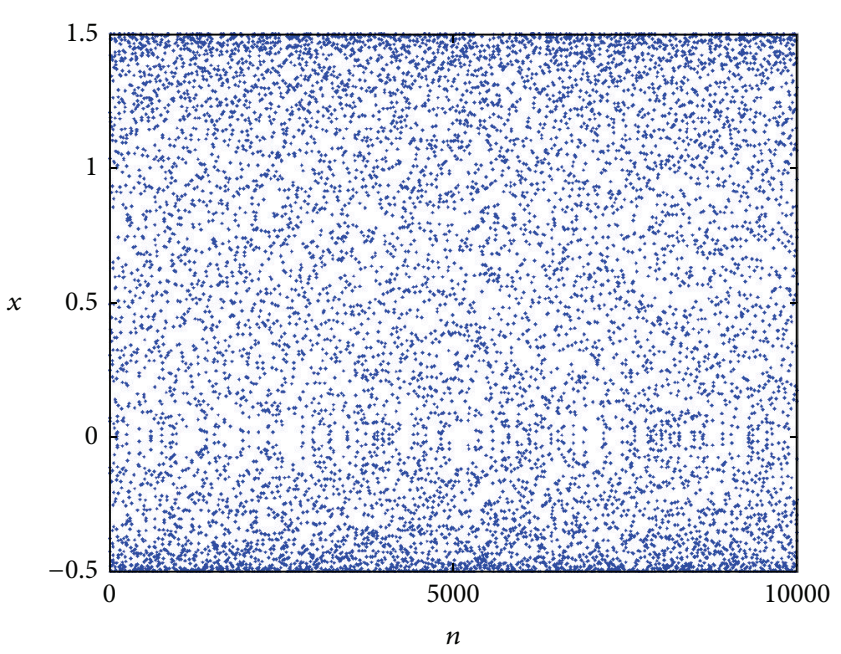

(b)

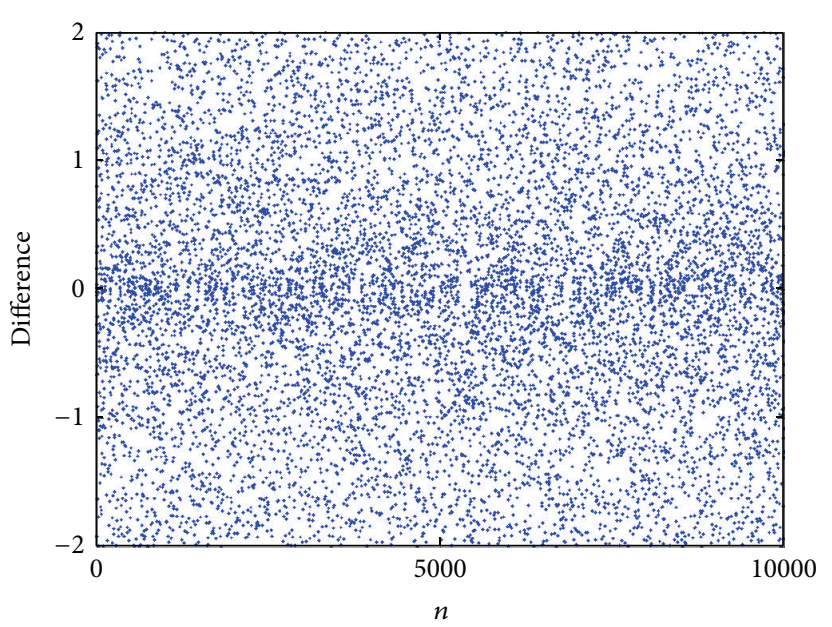

(c)

FIGURE 5: Sensitive dependence on initial point for mostly positive logistic map at $\lambda=2$ : (a) time waveform $x_{0}=0.05$, (b) time waveform $x_{0}=0.06$, and (c) initial point effect.

\subsubsection{Positive Logistic Map $f(x, \lambda, a, b)=\lambda x(a-b x)$}

(1) Range of $\lambda$. To ensure bounded output for all iterations, the value of $x$ should be limited to $x \in[0, a / b]$. The roots of the map, its critical point $x_{c}$, and its maximum value $x_{\max }$ are given by

$$
\begin{aligned}
f(x) & =0 \quad \text { for } x=0, \frac{a}{b}, \\
x_{c} & =\frac{a}{2 b} \\
f\left(x_{c}, \lambda_{\max }, a, b\right) & =\frac{a^{2} \lambda_{\max }}{4 b} \leq \frac{a}{b} \longrightarrow \lambda_{\max } \leq \frac{4}{a} .
\end{aligned}
$$

This inequality provides us with information on not only the maximum value of parameter $\lambda$ but also that of parameter $a$.
Therefore, the maximum values for the parameters of the map are

$$
\begin{aligned}
& \left(\lambda_{\max }, x_{\max }\right)=\left(\frac{4}{a}, \frac{a}{b}\right), \\
& a \in\left(0, a_{\max }\right], \text { where } a_{\max }=\frac{4}{\lambda} .
\end{aligned}
$$

(2) Fixed Points and Stability Condition. The fixed points are given by $x^{*}=f\left(x^{*}, \lambda, a, b\right)$; then

$$
\begin{aligned}
x^{*} & =\lambda x^{*}\left(a-b x^{*}\right), \\
x_{1}{ }^{*} & =0, \\
x_{2}{ }^{*} & =\frac{1}{b}\left(a-\frac{1}{\lambda}\right) .
\end{aligned}
$$

The study of the stability of the fixed points is based on the calculation of the absolute value of the first derivative with 
respect to $x$ at these points $\left|f^{\prime}\left(x^{*}, \lambda, a, b\right)\right|=\left|\lambda\left(a-2 b x^{*}\right)\right|$. They are either stable or unstable depending on whether this value is less or greater than "one," respectively. Otherwise, if the absolute value equals "one," then it is called a bifurcation point. The derivative of this map at the fixed points is given by

$$
\begin{aligned}
& f^{\prime}\left(x_{1}{ }^{*}, \lambda, b\right)=a \lambda, \\
& f^{\prime}\left(x_{2}{ }^{*}, \lambda, b\right)=2-a \lambda .
\end{aligned}
$$

Therefore, the values of $\lambda$ at which the system bifurcates and their function values are

$$
\begin{aligned}
\left|f^{\prime}\left(x^{*}, \lambda_{b}, a\right)\right| & =1 \longrightarrow \lambda_{b}=\frac{1}{a}, \frac{3}{a}, \\
f\left(x_{1}{ }^{*}, \lambda_{b 1}, a, b\right) & =0, \\
f\left(x_{2}{ }^{*}, \lambda_{b 2}, a, b\right) & =\frac{2 a}{3 b}, \\
\left(x_{b}, \lambda_{b}\right) & =\left\{\left(0, \frac{1}{a}\right),\left(\frac{2 a}{3 b}, \frac{3}{a}\right)\right\} .
\end{aligned}
$$

\subsubsection{Mostly Positive Logistic Map $f(x, \lambda, a, b)=-\lambda x(a-b x)$}

(1) Range of $\lambda$. Similarly, for mostly positive logistic map, the solution should be limited to $x \in[-a /(2 b), 3 a /(2 b)]$,

$$
\begin{gathered}
x_{c}=\frac{a}{2 b}, \\
f\left(x_{c}, \lambda_{\text {max }}, a, b\right)=-\frac{a^{2} \lambda_{\text {max }}}{4 b} \geq-\frac{a}{2 b}, \\
\left(\lambda_{\text {max }}, x_{\text {min }}, x_{\text {max }}\right)=\left(\frac{2}{a},-\frac{a}{2 b}, \frac{3 a}{2 b}\right), \\
a \in\left(0, a_{\text {max }}\right], \text { where } a_{\max }=\frac{2}{\lambda} .
\end{gathered}
$$

(2) Fixed Points and Stability Condition. Similarly, for mostly positive logistic map, the value of $\lambda$ at which the system bifurcates and the corresponding function value are

$$
\left(x_{b}, \lambda_{b}\right)=\left(0, \frac{1}{a}\right)
$$

3.2. Vertical Scaling $x_{n+1}= \pm \lambda x_{n}\left(1 \pm b x_{n}\right)$. In this map, an extra parameter $b$ is added which affects the vertical scaling of the bifurcation diagram; that is, it is a special case of the independent scaling map in which $a=1$. This map could be used to control the vertical axis, that is, the values of the output, not the bifurcation points. In this subsection, the range of $\lambda$, fixed points and their stability analysis, and behavior dependence on different system parameters for the proposed maps are discussed.

\subsubsection{Positive Logistic Map $f(x, \lambda, b)=\lambda x(1-b x)$}

(1) Range of $\lambda$. Substituting $a=1$ in (17a) yields

$$
\left(\lambda_{\max }, x_{\max }\right)=\left(4, \frac{1}{b}\right) .
$$

The function iterations $f^{m}(x, \lambda, b)$ at $b=2$ are shown in Figure 6 , where the maximum value of the output $x_{\max }=$ $1 / b=0.5$ and the parameter $b$ affects the vertical axis only. The number of peaks increases as $m$ increases and as $\lambda$ approaches its maximum value $\lambda_{\max }=4$ which exhibits maximum chaotic behavior. On the other hand, for $\lambda<3$, no peaks could be noticed even in higher iterations.

(2) Fixed Points and Stability Condition. Substituting $a=1$ in (20c) yields

$$
\left(x_{b}, \lambda_{b}\right)=\left\{(0,1),\left(\frac{2}{3 b}, 3\right)\right\} .
$$

(3) Steady State Solutions versus System Parameters. Figure 7 shows the steady state solutions versus both system parameters $\lambda$ and $b$ for vertical scaling positive logistic map. The bifurcation diagram versus $\lambda$ exhibits similar behavior to the conventional case but with vertical scaling by the factor $(1 / b)$ as shown in Figure 7(a). The nontrivial solution appears once $\lambda$ exceeds the first bifurcation point $\lambda_{b 1}=1$, while the second bifurcation point is $\lambda_{b 2}=3$ with corresponding steady state value $x_{b 2}=2 /(3 b)$. Figure $7(\mathrm{a})$ also shows the Cobweb plot of the map at $\lambda_{\max }=4$ and $b=10$, which differs as the parameters change. The Cobweb is a rough plot for an orbit of $x$, where the graph of the map function is sketched together with the diagonal line $y=x$. The plot shows that the map exhibits chaotic behavior, since the orbit has a nonperiodic sequence and generates multiple outputs that cover the whole range of $x$.

Figure 7(b) shows the behavior dependence on $b$ for a fixed value of $\lambda$. Theoretically, the only condition on $b$ is to be positive. It should be noted that for larger values of $b$, extremely small initial points are needed such that they belong to the allowable range. Disregarding this note would yield results that might be falsely interpreted as a case of instability. Plotting starts at $b=0.1$ to avoid larger values of $x$ that decrease the clarity of the figure. The range of output results $x$ depends on $b$; that is, $x \in(0,1 / b)$, as proposed by our previous analysis. The type of solution is either fixed, period2 , multi-periods, or chaos depending on the value of $\lambda$. As $\lambda$ increases, the response covers more values belonging to the allowable range until it covers the whole range $x \in(0,1 / b)$ at $\lambda_{\text {max }}=4$.

3.2.2. Mostly Positive Logistic Map $f(x, \lambda, b)=-\lambda x(1-b x)$

(1) Range of $\lambda$. Substituting $a=1$ in (21b) yields

$$
\left(\lambda_{\max }, x_{\min }, x_{\max }\right)=\left(2,-\frac{1}{2 b}, \frac{3}{2 b}\right) .
$$



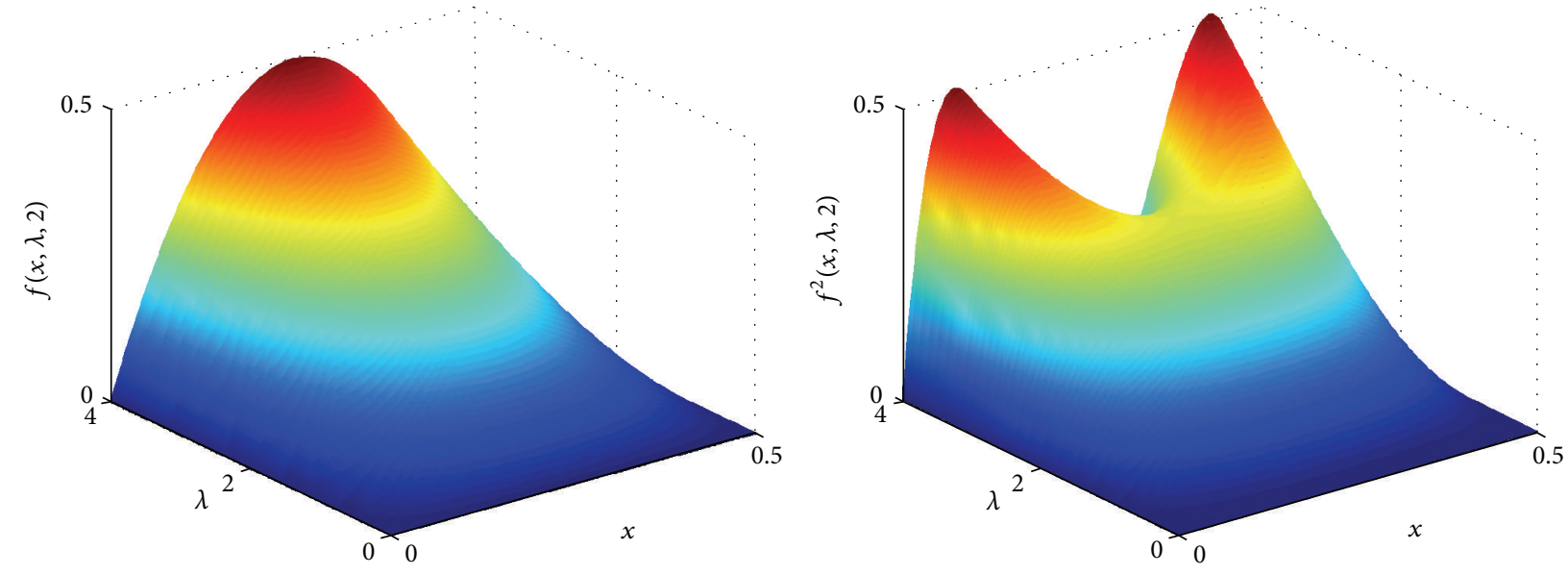

(a)
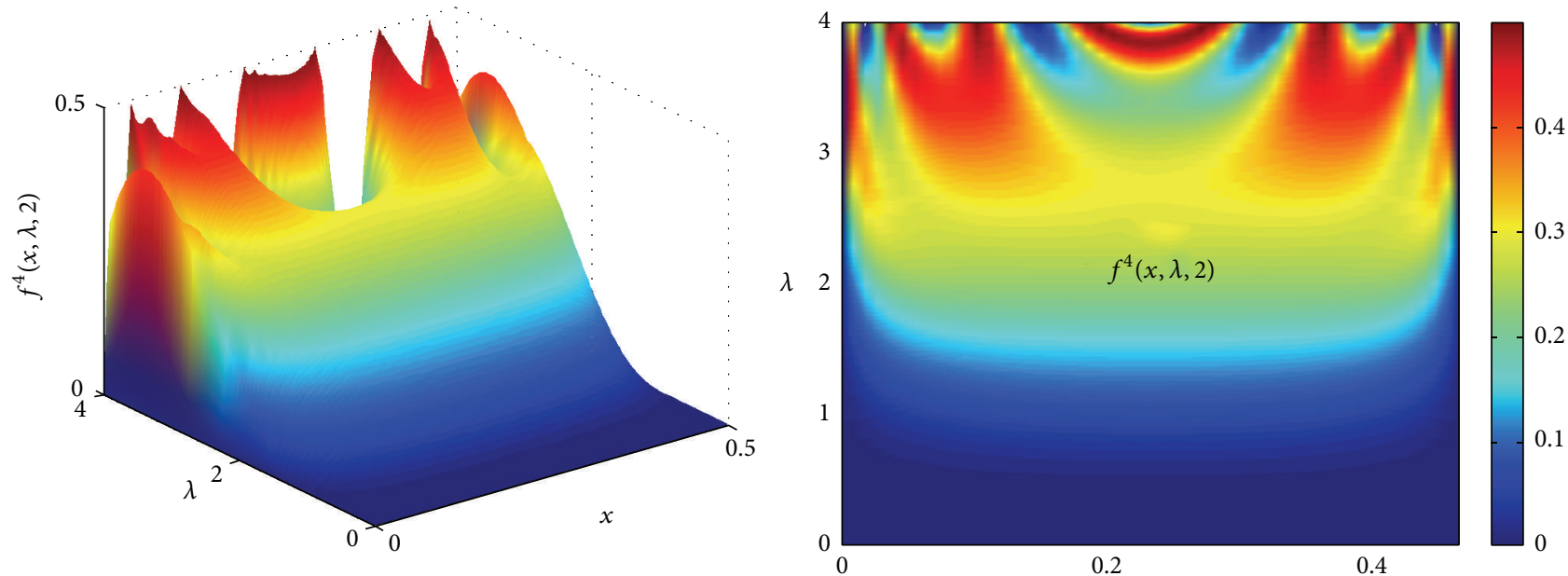

(b)
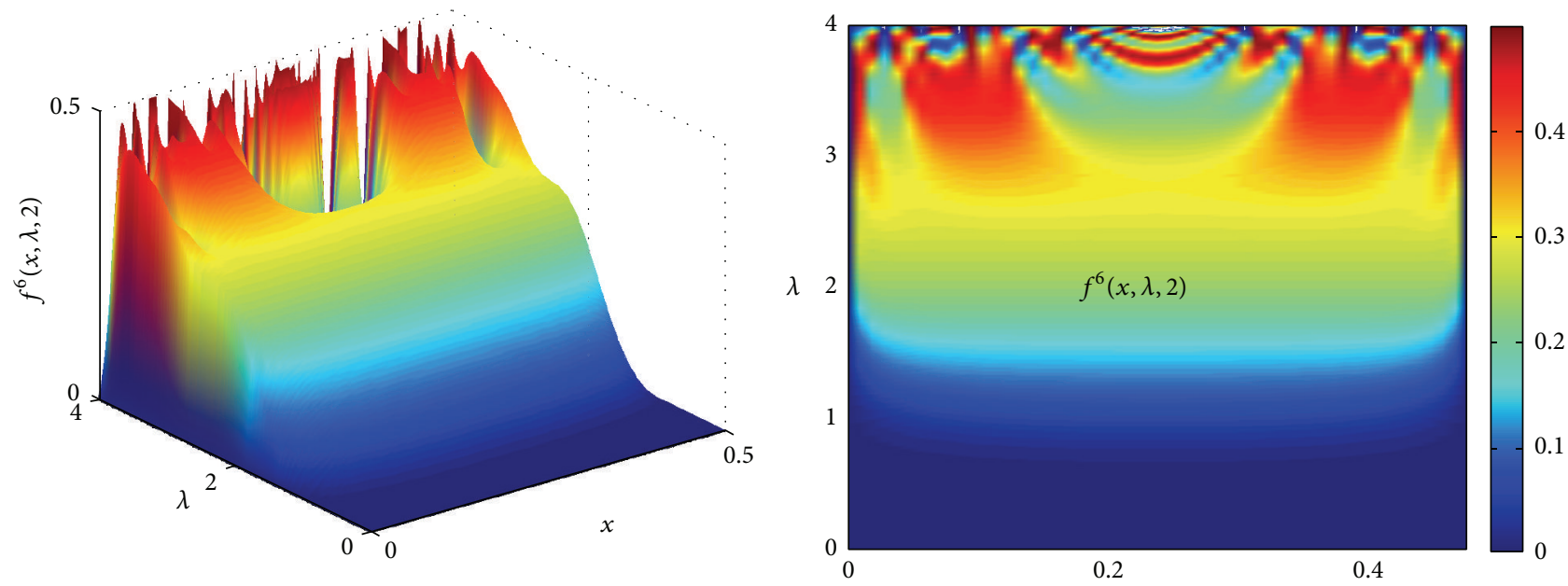

(c)

FIGURE 6: Function iterations of vertical scaling positive logistic map $f^{m}(x, \lambda, b)$ at $b=2$ for $m=\{1,2,4,6\}$. 

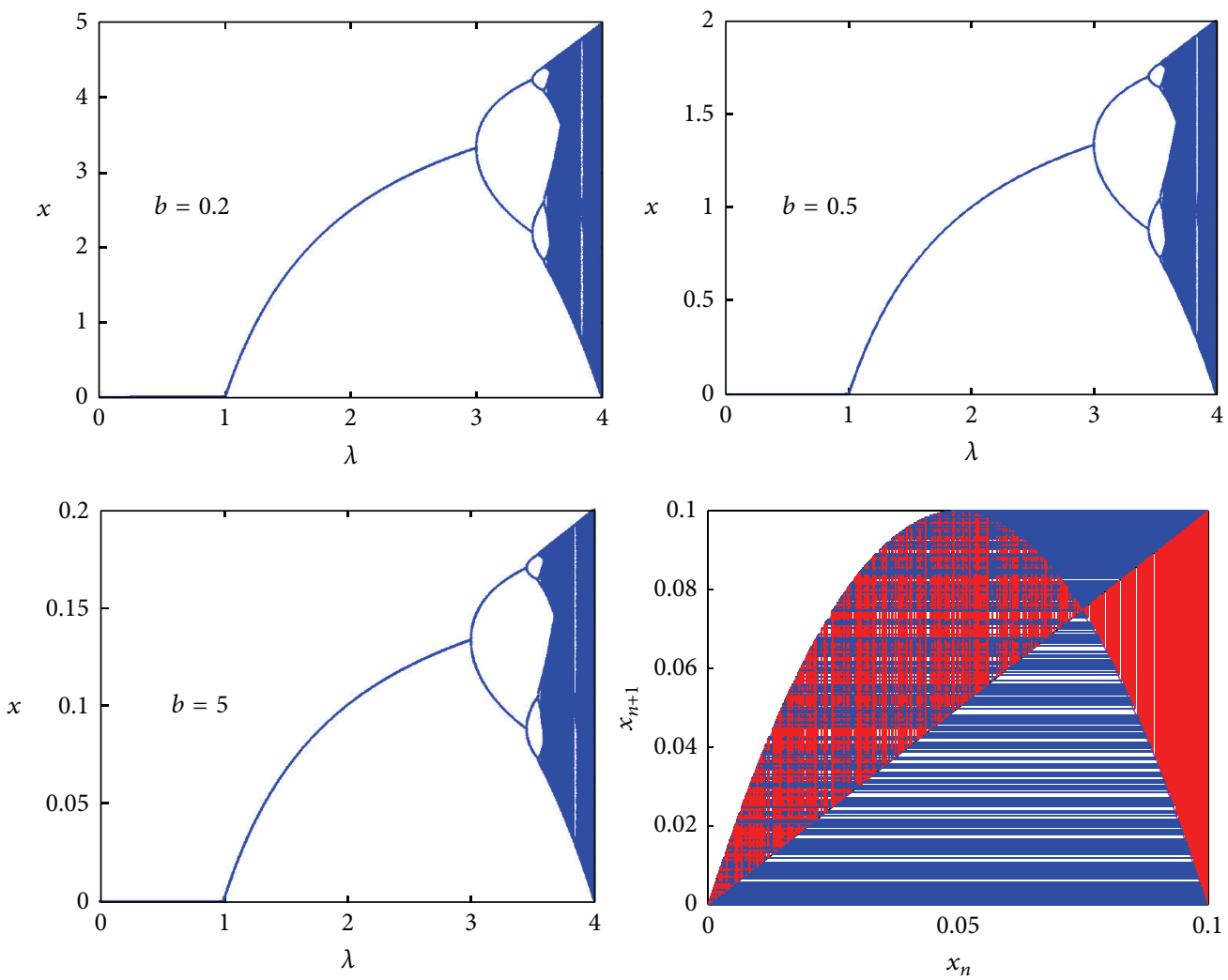

(a)
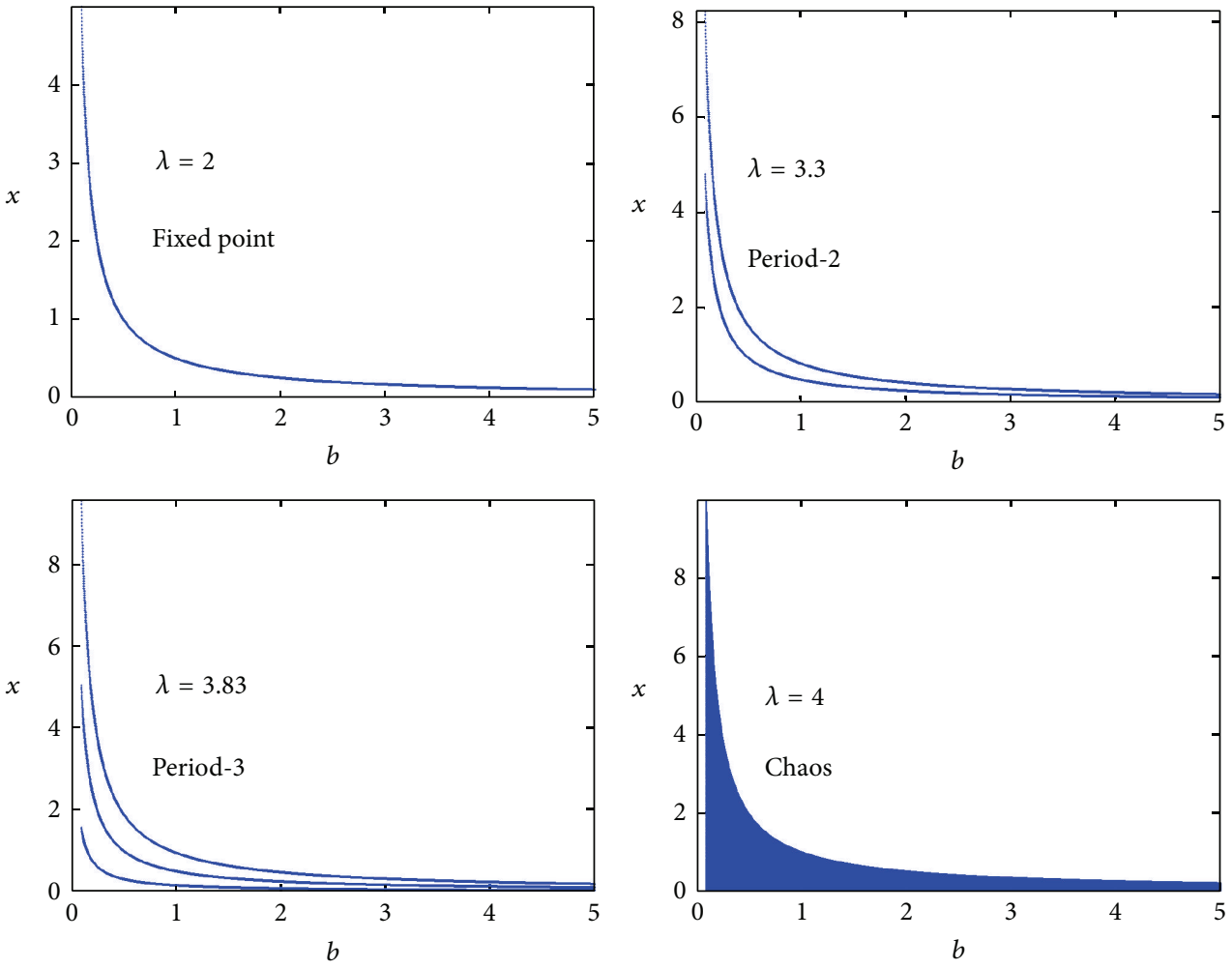

(b)

Figure 7: (a) Bifurcation diagram versus $\lambda$ for different values of $b=\{0.2,0.5,5\}$ and Cobweb plot at $\lambda=4, b=10$. (b) Steady state solutions of $x$ versus $b$ for different values of $\lambda=\{2,3.3,3.83,4\}$ for vertical scaling positive logistic map. 
(2) Fixed Points and Stability Condition. Substituting $a=1$ in (22) yields

$$
\left(x_{b}, \lambda_{b}\right)=(0,1) .
$$

(3) Steady State Solutions versus System Parameters. Figure 8 shows the steady state solutions versus both system parameters $\lambda$ and $b$ and the Cobweb plot at $\lambda_{\max }=2$ and $b=$ 10 for mostly positive logistic map. The results could be interpreted and proved to be conforming to our analysis. Ten different snapshots of the bifurcation diagram versus the system parameter $\lambda$ for both maps are shown in Figure 9, where the idea of vertical scaling is quite clear.

3.2.3. Maximum Lyapunov Exponent. From the nonlinear analysis of chaos, the maximum Lyapunov exponent MLE should be positive to prove chaotic behavior. MLE is one of the basic properties of chaotic systems and it is given by [29]

$$
\text { MLE }=\lim _{n \rightarrow \infty}\left(\frac{1}{n} \sum_{i=0}^{n-1} \ln \left|f^{\prime}\left(x_{i}\right)\right|\right)
$$

for a discrete map $f(x)$ and the orbit starting at $x_{0}$, where $\ln$ is the natural logarithm.

For both vertical scaling maps, the time evolution of MLE for different values of $b$ roughly reaches the same steady state value. This constant value is the same as that of the conventional map MLE $=\ln 2$. Besides, all map variations exhibit the same value at $\lambda_{\max }$ representing maximum chaotic behavior. This value has been proved for almost all orbits of chaotic maps, starting from the tent map and extending to the logistic map and others by conjugacy of maps [29]. Figure 10(a) shows 3D plot of MLE as a function of both $\lambda$ and $b$, in addition to the corresponding contour plot, for vertical scaling positive logistic map, while Figure 10(b) shows them for vertical scaling mostly positive logistic map.

3.3. Zooming $x_{n+1}= \pm \lambda x_{n}\left(a \pm x_{n}\right)$. In this map, an extra parameter $a$ is added which affects the zooming of the bifurcation diagram; that is, it is a special case of the independent scaling map in which $b=1$. Moreover, there is a new bifurcation diagram presented with respect to the system parameter $a$ that will be generalized later on. This map is richer in analysis and could be used for controlling both axes, that is, the bifurcation points in addition to the corresponding output values. This control can be achieved only in a dependent way, such that the area of the bifurcation diagram versus $\lambda$ remains constant. In this subsection, the range of $\lambda$, fixed points and their stability analysis, and two different bifurcation diagrams are discussed.

\subsubsection{Positive Logistic Map $f(x, \lambda, a)=\lambda x(a-x)$}

(1) Range of $\lambda$. Substituting $b=1$ in (17a) yields

$$
\begin{aligned}
& \left(\lambda_{\max }, x_{\max }\right)=\left(\frac{4}{a}, a\right), \\
& a \in\left(0, a_{\max }\right], \text { where } a_{\max }=\frac{4}{\lambda} .
\end{aligned}
$$

(2) Fixed Points and Stability Condition. Substituting $b=1$ in (20c) yields

$$
\left(x_{b}, \lambda_{b}\right)=\left\{\left(0, \frac{1}{a}\right),\left(\frac{2 a}{3}, \frac{3}{a}\right)\right\} .
$$

(3) Steady State Solutions versus System Parameters. Figure 11(a) shows the bifurcation diagram versus $\lambda$ for different values of $a$ for zooming positive logistic map. The horizontal and vertical axes corresponding to the values of the parameter $\lambda$ and the solution $x$ are scaled by $(1 / a)$ and $a$, respectively, so that the total area is still the same and equals a constant value of $a(4 / a)=4$ square units. Figure 11(a) also shows the Cobweb plot at $a=4$ and $\lambda=1$, which exhibits chaotic behavior where the whole range of $x$ is covered.

Figure 11(b) shows the bifurcation diagram versus $a$ for different values of $\lambda$, where the diagram has equal axes lengths which we will call a square-axis, and its area is a function of $\lambda$. The unity area square results in the case of $\lambda=4$ which corresponds to the maximum value of $\lambda$ in the unity scaling case $(a=1)$. Recalling that $a_{\max }=(4 / \lambda)$ as given by $(28 \mathrm{~b})$, then the values of $a$ are scaled by $(4 / \lambda)$. Accordingly, the values of $x$ undergo the same scaling and $x \in[0,4 / \lambda]$. Thus, for positive logistic map, the horizontal and vertical axes are scaled together by the same ratio which equals $(4 / \lambda)$.

\subsubsection{Mostly Positive Logistic Map $f(x, \lambda, a)=-\lambda x(a-x)$}

(1) Range of $\lambda$. Substituting $b=1$ in (21b) yields

$$
\begin{aligned}
& \left(\lambda_{\max }, x_{\min }, x_{\max }\right)=\left(\frac{2}{a},-\frac{a}{2}, \frac{3 a}{2}\right), \\
& a \in\left(0, a_{\max }\right], \text { where } a_{\max }=\frac{2}{\lambda} .
\end{aligned}
$$

(2) Fixed Points and Stability Condition. Substituting $b=1$ in (22) yields

$$
\left(x_{b}, \lambda_{b}\right)=\left(0, \frac{1}{a}\right)
$$

(3) Steady State Solutions versus System Parameters. Figure 12(a) shows the bifurcation diagram versus $\lambda$ for different values of $a$ for zooming mostly positive logistic map. The diagrams are quite similar to those in the case of zooming positive logistic map in having constant area axes. Figure 12(a) also shows the Cobweb plot at $a=4$ and $\lambda=0.5$, which shows chaotic behavior where the whole range of $x$ is covered.

Figure 12(b) shows the bifurcation diagram versus $a$ for different values of $\lambda$ for mostly positive map. Similar to positive map, the horizontal and vertical axes are scaled together by the same ratio which equals $(2 / \lambda)$ for mostly positive map. Yet, they do not form a square-axis due to its alternating sign nature, where $x \in[-1 / \lambda, 3 / \lambda]$. Instead, the length of the vertical axis is double that of the horizontal axis but extends to the negative part asymmetrically. Therefore, the bifurcation diagrams with respect to $\lambda$ are of equal area for both zooming maps. However, the bifurcation diagrams 

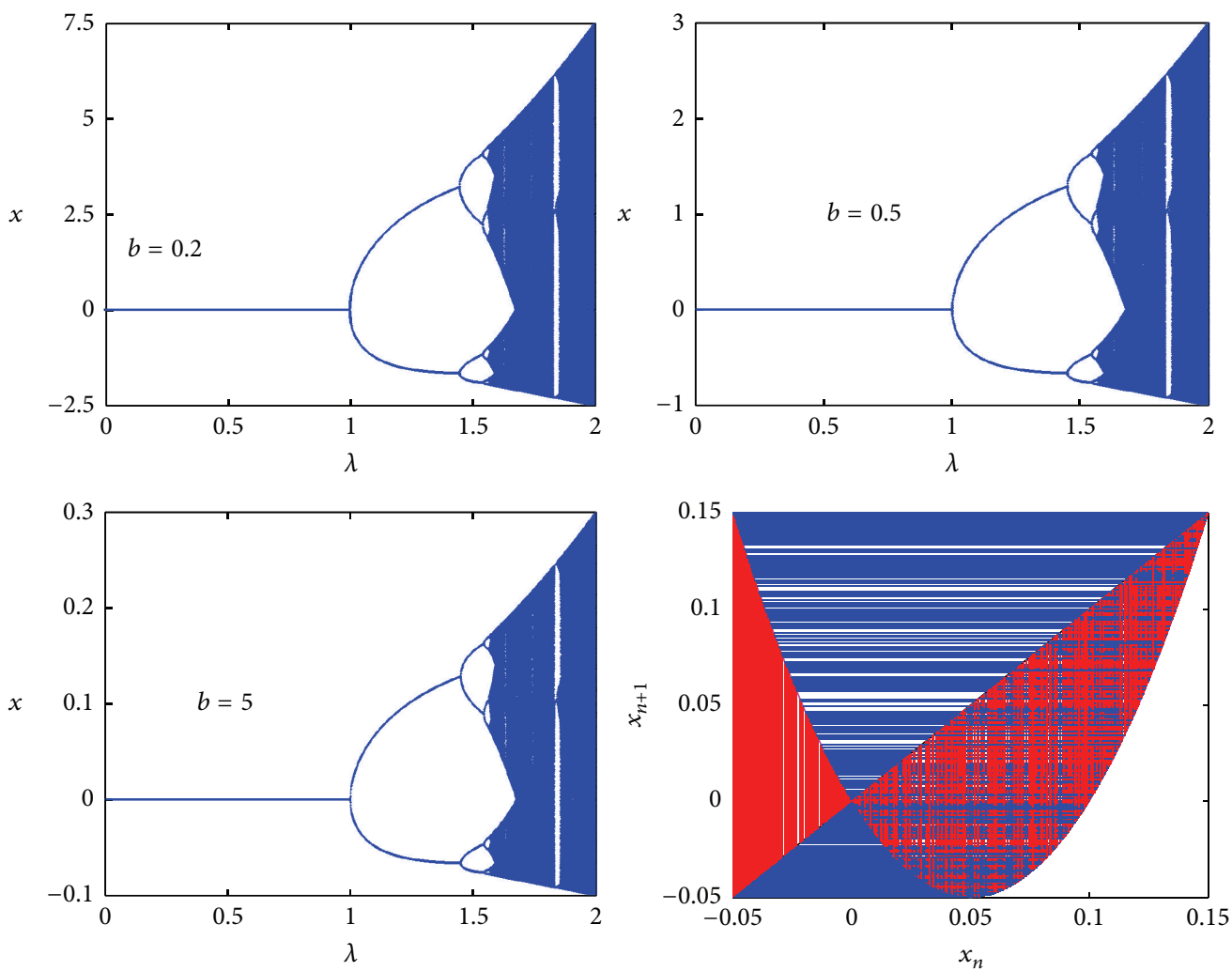

(a)
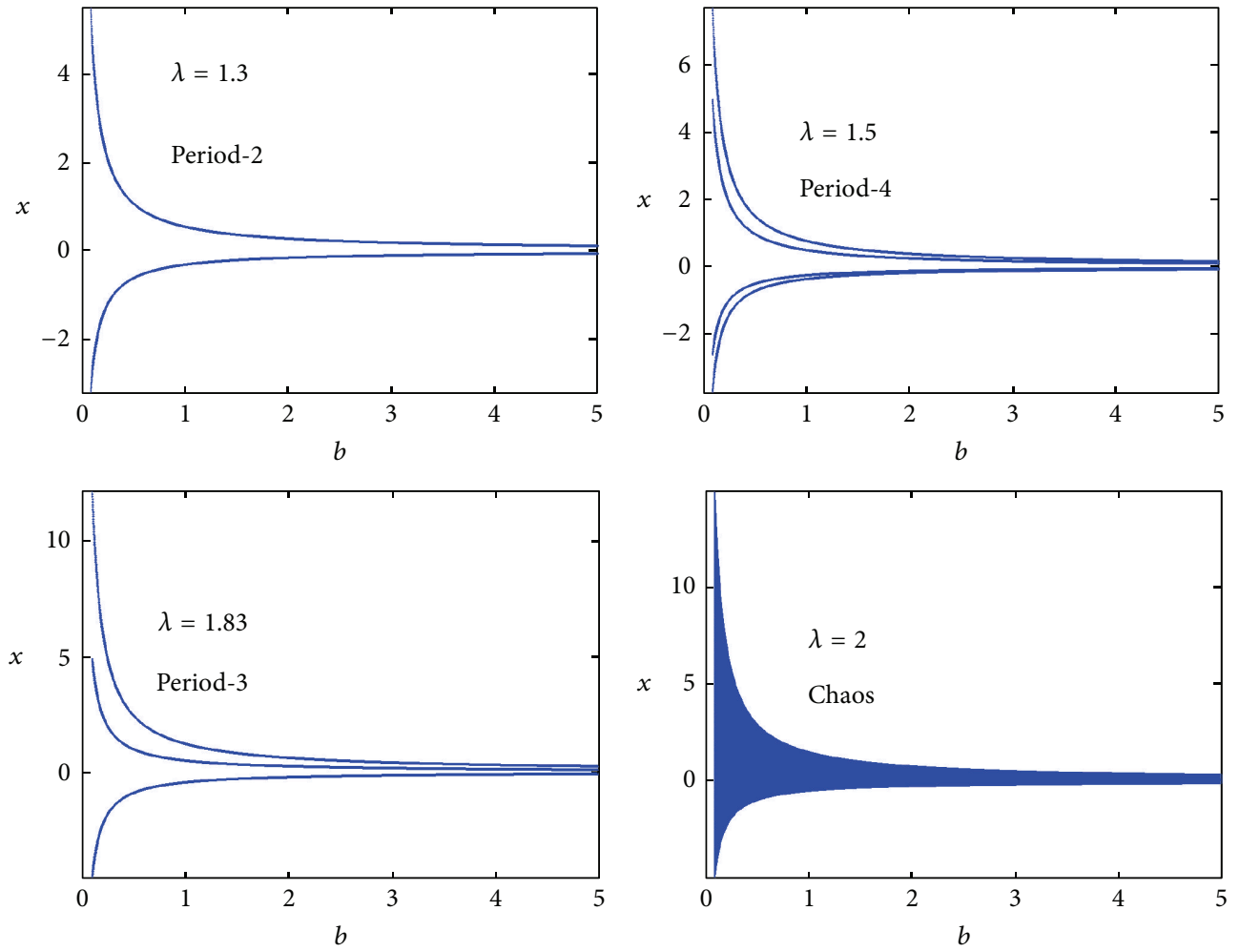

(b)

FIGURE 8: (a) Bifurcation diagram versus $\lambda$ for different values of $b=\{0.2,0.5,5\}$ and Cobweb plot at $\lambda=2, b=10$. (b) Steady state solutions of $x$ versus $b$ for different values of $\lambda=\{1.3,1.5,1.83,2\}$ for vertical scaling mostly positive logistic map. 


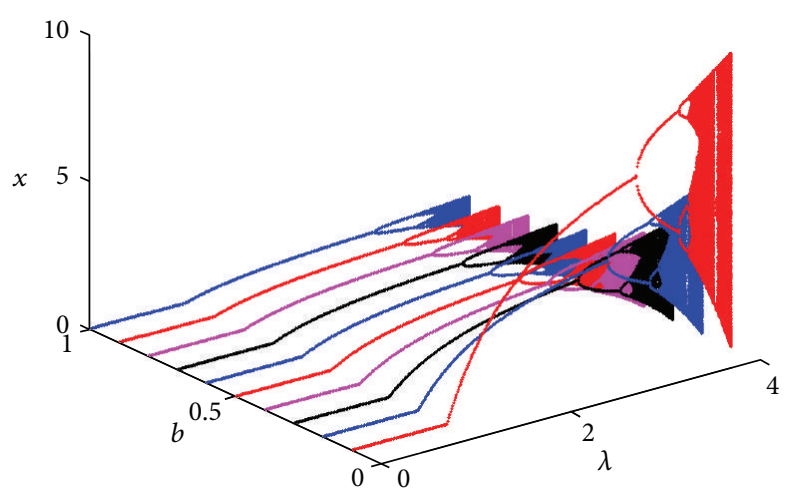

(a)

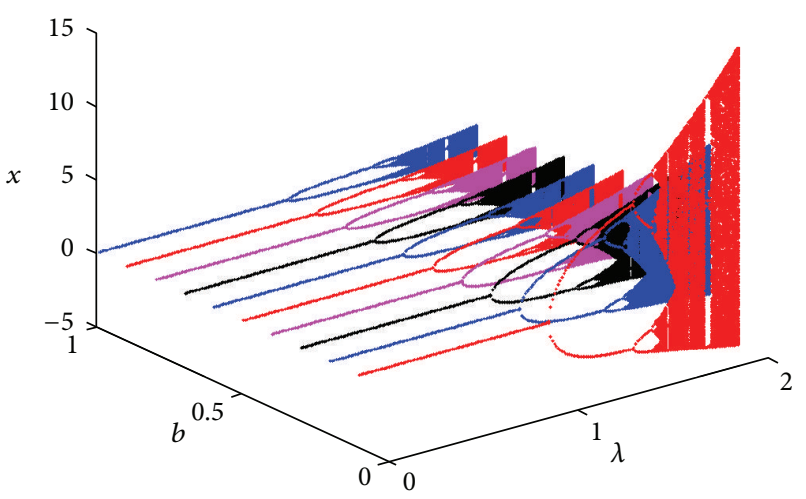

(b)

FIGURE 9: Ten different snapshots of the bifurcation diagram versus $\lambda$ for vertical scaling (a) positive and (b) mostly positive maps at $b=$ $\{0.1,0.2, \ldots, 1\}$.
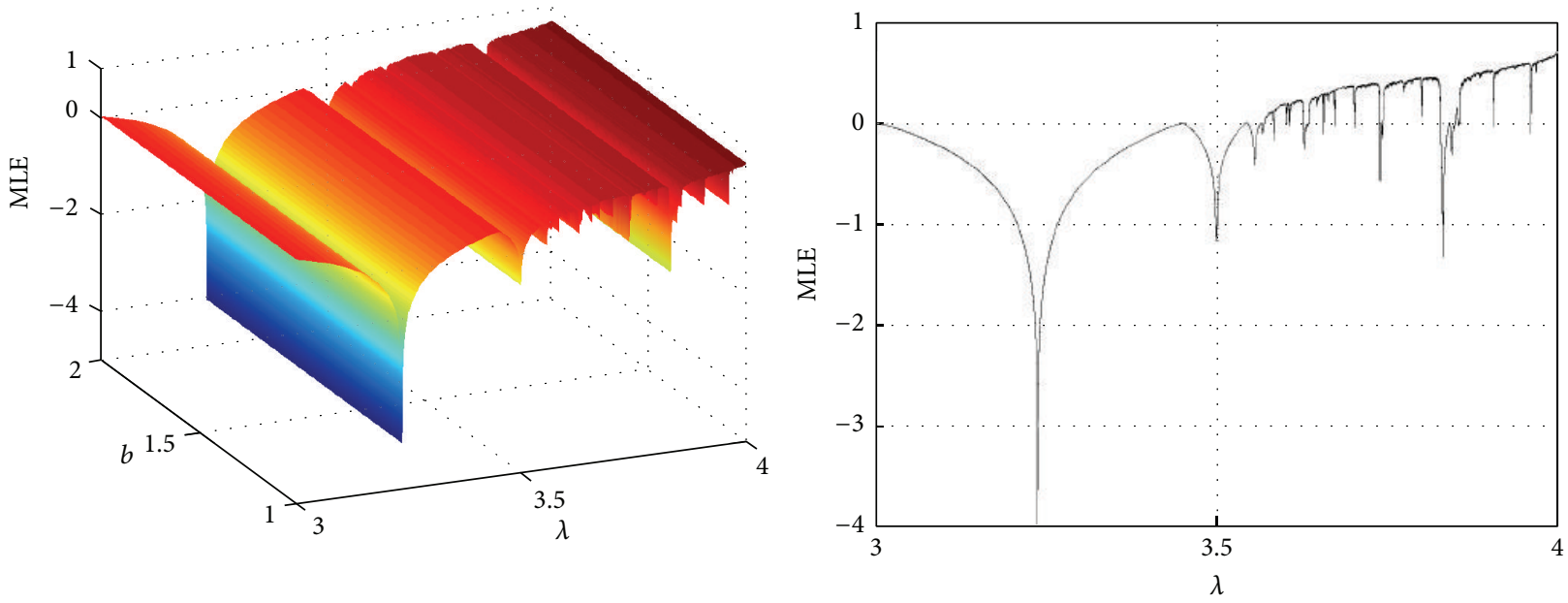

(a)
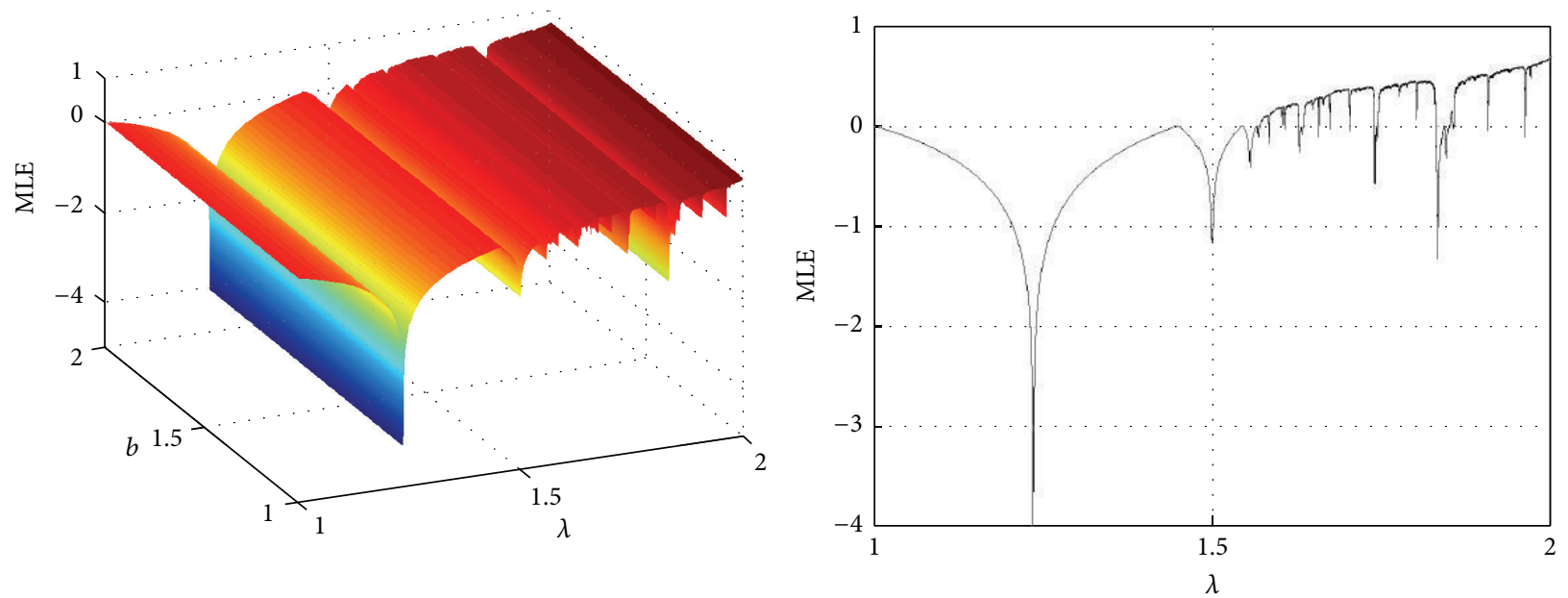

(b)

Figure 10: MLE as a function of both $\lambda$ and $b$ for vertical scaling (a) positive and (b) mostly positive logistic maps. 

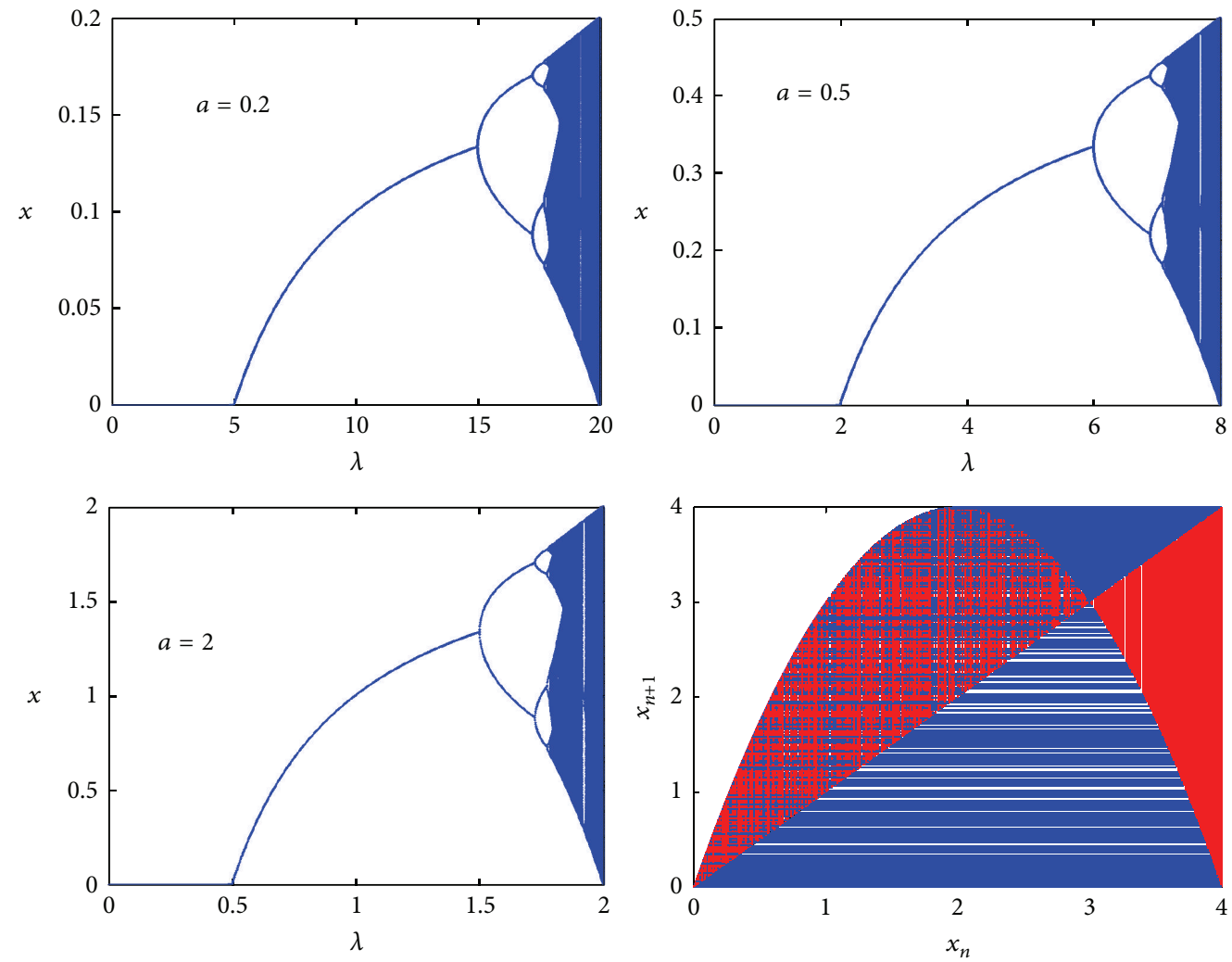

(a)
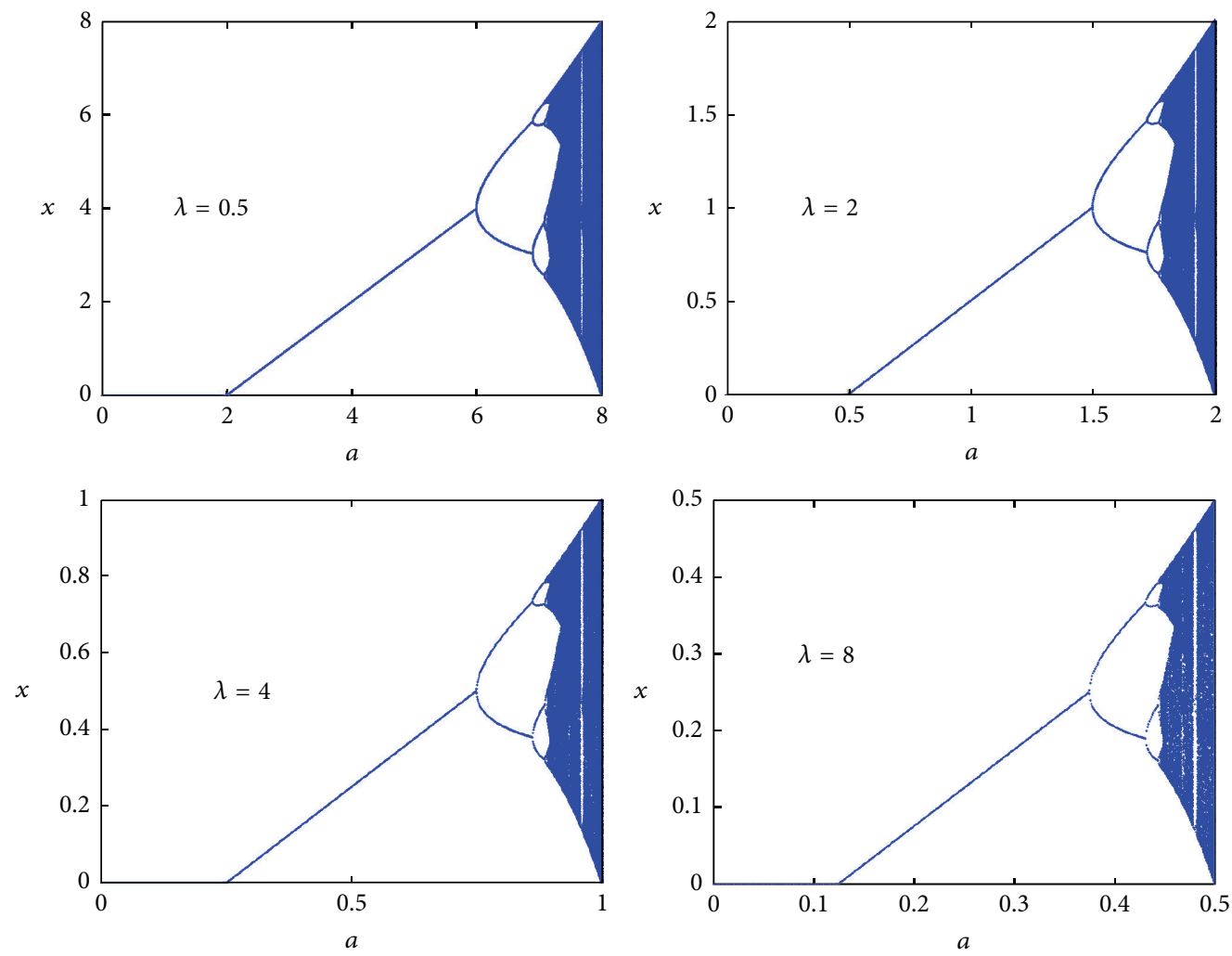

(b)

FIGURE 11: (a) Bifurcation diagram versus $\lambda$ for different values of $a=\{0.2,0.5,2\}$ and Cobweb plot at $a=4, \lambda=1$. (b) Bifurcation diagram versus $a$ for different values of $\lambda=\{0.5,2,4,8\}$ for zooming positive logistic map. 

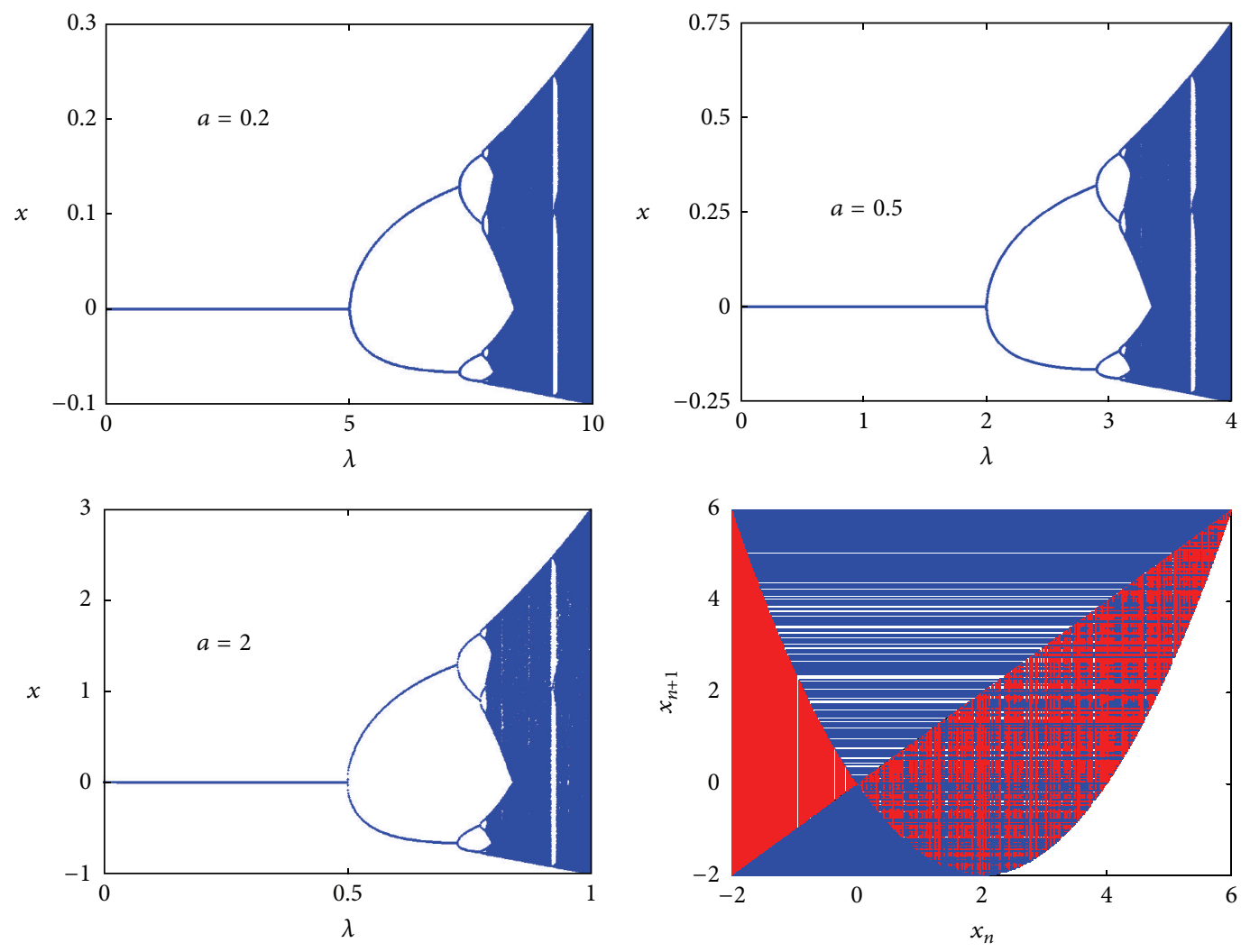

(a)
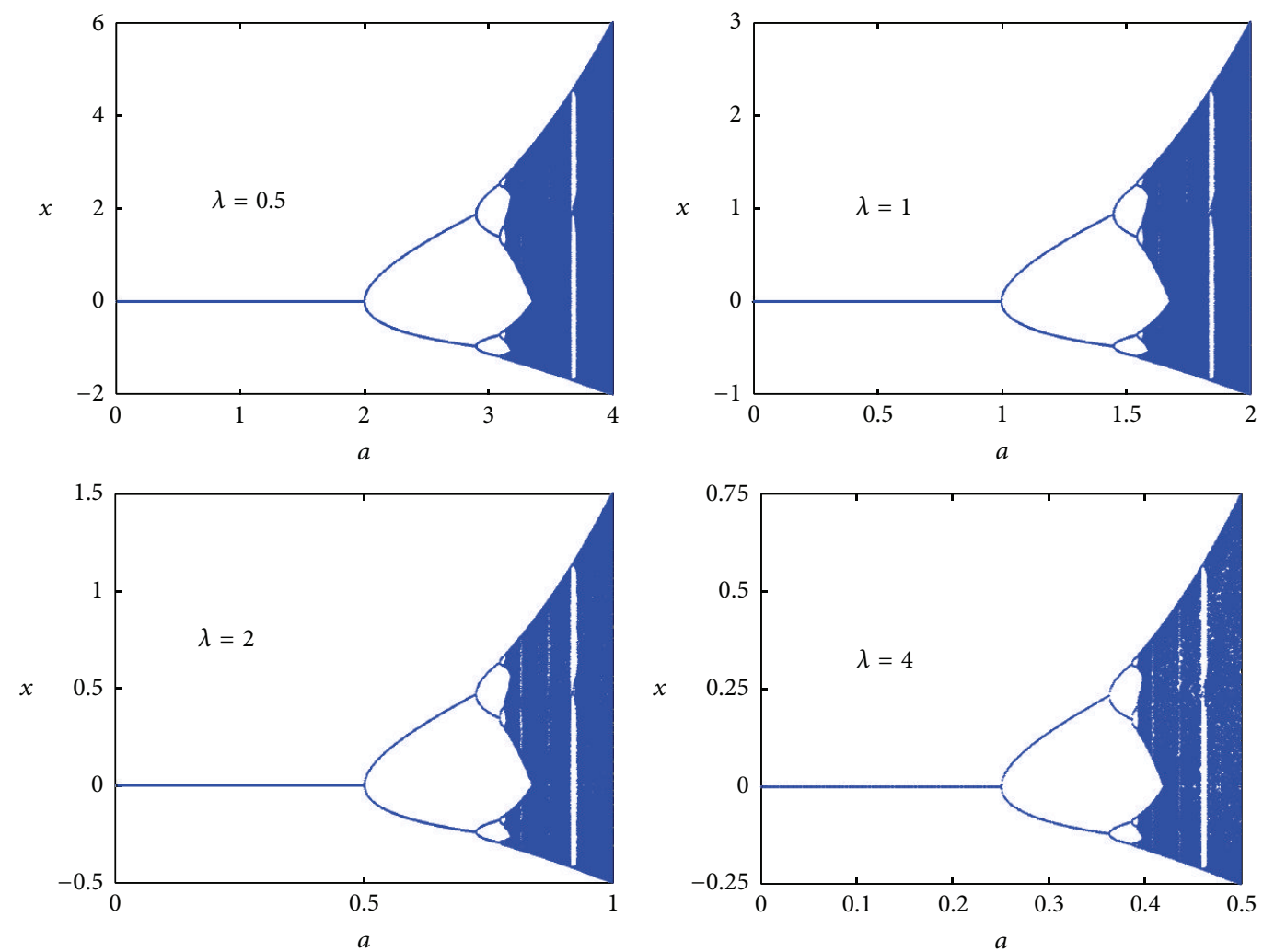

(b)

FIgURE 12: (a) Bifurcation diagram versus $\lambda$ for different values of $a=\{0.2,0.5,2\}$ and Cobweb plot at $a=4, \lambda=0.5$. (b) Bifurcation diagram versus $a$ for different values of $\lambda=\{0.5,1,2,4\}$ for zooming mostly positive logistic map. 


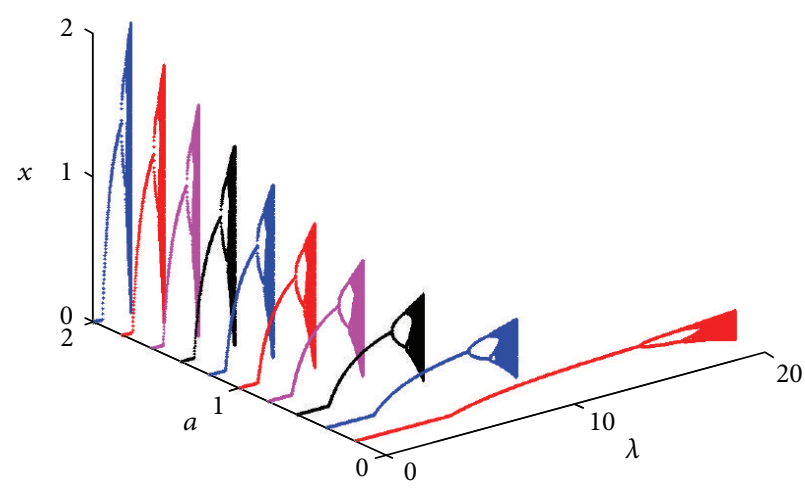

(a)

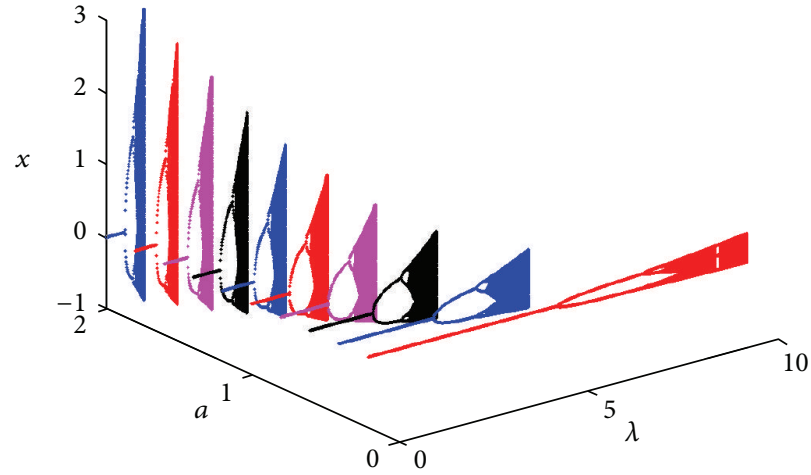

(b)

Figure 13: Ten different snapshots of the bifurcation diagram versus $\lambda$ for zooming (a) positive and (b) mostly positive maps at $a=$ $\{0.2,0.4, \ldots, 2\}$.

with respect to $a$ are of equal axes for the positive map and a vertical axis double the horizontal axis for the mostly positive map. This enriches the map characteristics and raises its degrees of freedom to be used in control applications according to the required constraints.

Figure 13 shows snapshots of the bifurcation diagrams versus $\lambda$ at different values of $a$ for both zooming maps. Figure 14 shows the other type of bifurcation diagram which is plotted versus $a$ at different values of $\lambda$ for both maps. The snapshots reflect the inverse proportionality relation between $\lambda$ and $a$, where as $a$ increases, the value of $\lambda_{\max }$ decreases and vice versa. Similarly, as $\lambda$ increases, the value of $a_{\max }$ decreases and vice versa.

3.3.3. Maximum Lyapunov Exponent. Figure 15(a) shows 3D plot of MLE as a function of both $\lambda$ and $a$ for zooming positive logistic map, while Figure 15(b) shows it for zooming mostly positive logistic map. This continuous surface plot illustrates the dependence of the allowed range of $\lambda$ on the value of $a$ for both zooming positive and mostly positive logistic maps. This fact is further indicated by discrete snapshots of MLE as a function of $\lambda$ for different values of $a$. However, the value of MLE remains the same for maximum chaotic behavior, that is, at $\lambda_{\max }$. The same steady state value MLE $=\ln 2$ is obtained which equals that of the conventional logistic map as previously explained.

\section{General Schematic of the Bifurcation Diagrams}

Recalling the analyses and discussions throughout this paper and combining them with the results obtained in the last section, we can sketch a generalized schematic for the bifurcation diagrams. The key points of the two different bifurcation diagrams versus the system parameters $\lambda$ and $a$ for both independent scaling positive and mostly positive maps can be summarized as follows.

\subsection{Positive Logistic Map $x_{n+1}=\lambda x_{n}\left(a-b x_{n}\right)$}

4.1.1. Bifurcation Diagram versus $\lambda$. The values of $\lambda_{b}$ for the first and second bifurcation points are (1/4) and (3/4) of $\lambda_{\text {max }}$, respectively, where $\lambda_{\text {max }}=4 / a$. Moreover, the value of the first nontrivial bifurcation point $x_{b 2}$ equals $(2 / 3)$ of the maximum value $x_{\max }$, where $x_{\max }=a / b$. Figure 16(a) shows the key points of the bifurcation diagram versus $\lambda$ in terms of $a$ and $b$ which conform to the results expected by our analysis.

4.1.2. Bifurcation Diagram versus $a$. From another point of view, we can design the bifurcation diagram with respect to the parameter $a$ where the bifurcation points and maximum values are shown in Figure 16(b) in terms of the other parameters $b$ and $\lambda$. The values of $a$ at which bifurcation occurs $a_{b}$ are (1/4) and (3/4) of $a_{\max }$, where $a_{\max }=4 / \lambda$. Moreover, the value of the first nontrivial bifurcation point $x_{b 2}$ equals $(1 / 2)$ the maximum value $x_{\max }$, where $x_{\max }=$ $4 /(\lambda b)$.

\subsection{Mostly Positive Logistic Map $x_{n+1}=-\lambda x_{n}\left(a-b x_{n}\right)$}

4.2.1. Bifurcation Diagram versus $\lambda$. Figure 17 (a) shows the key points of the bifurcation diagram versus $\lambda$ in terms of $a$ and $b$. The value of $\lambda_{b}$ is $(1 / 2)$ of $\lambda_{\max }$, where $\lambda_{\max }=2 / a$. The minimum and maximum values of $x$ are $x_{\min }=-a /(2 b)$ and $x_{\max }=(3 a) /(2 b)$, respectively.

4.2.2. Bifurcation Diagram versus a. Figure 17(b) shows the key points of the bifurcation diagram versus $a$ in terms of $b$ and $\lambda$. The value of $a_{b}$ is $(1 / 2)$ of $a_{\max }$, where $a_{\max }=2 / \lambda$. The minimum and maximum values of $x$ are $x_{\min }=-1 /(\lambda b)$ and $x_{\max }=3 /(\lambda b)$, respectively.

\section{Logistic Map Design Procedure}

Table 2 summarizes the results of independent scaling positive and mostly positive logistic maps. As previously detailed, the other two maps exhibit the same properties except for values of $x$ that have the same magnitude, but opposite sign. 


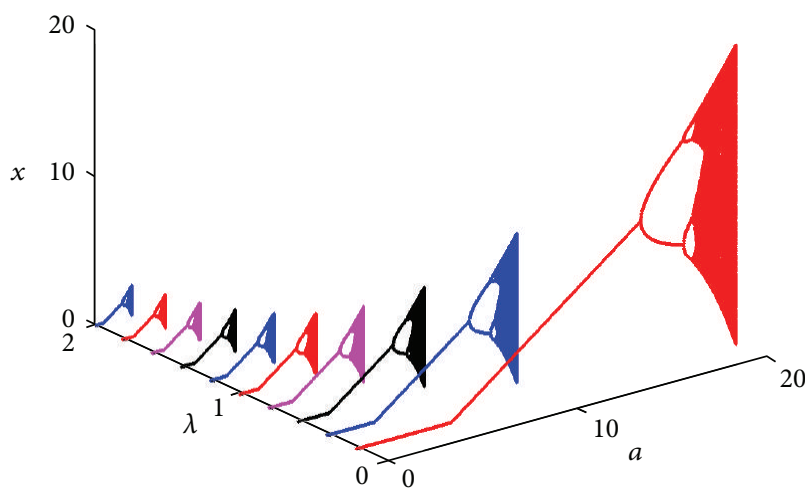

(a)

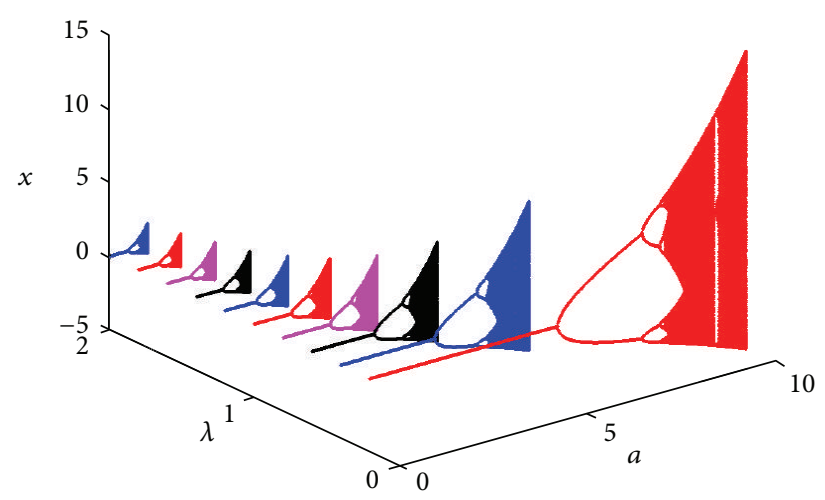

(b)

FIGURE 14: Ten different snapshots of the bifurcation diagram versus $a$ for zooming (a) positive and (b) mostly positive maps at $\lambda=$ $\{0.2,0.4, \ldots, 2\}$.

TABLE 2: Comparison between the main aspects of the proposed generalized logistic maps.

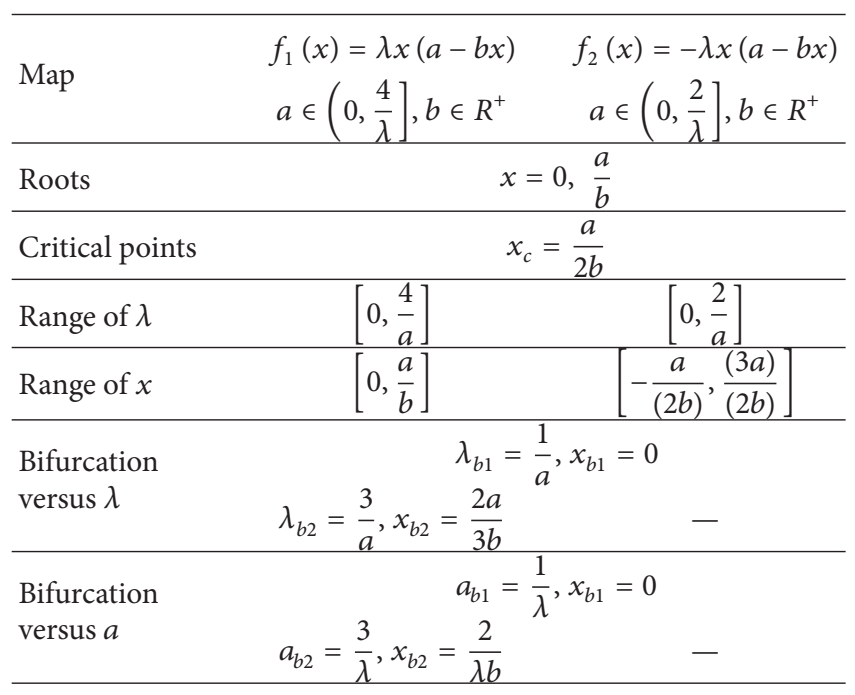

Consequently, any required scaling can be achieved using the two extra parameters $a$ and $b$, for bifurcation diagram versus $\lambda$. The horizontal scaling can be controlled by the parameter $a$, while the vertical scaling depends on the value $(a / b)$. To achieve horizontal scaling only while keeping the vertical axis with the values corresponding to the unity scaling case, the value of $a$ should equal that of $b$. The bifurcation diagram versus $a$ could be similarly designed and controlled by the values of $\lambda$ and $b$. The remarkable advantage in the proposed variations on the discrete $1 \mathrm{D}$ logistic map is not only about scaling or shifting but also about the increased complexity of the output that makes it more unpredictable, that is, more convenient for applications that employ chaos.

5.1. Design Examples. The required specifications on the bifurcation diagram could be realized with respect to one of the parameters $\lambda$ and $a$. Both realizations are considered; we denote the value of the parameter at which first, or second, bifurcation occurs as $\operatorname{par}_{b 1}$ and $\operatorname{par}_{b 2}$, respectively, and the maximum value of the parameter as par $_{\max }$; where par could be $\lambda$ or $a$. Let us also define extrema $(x)$ such that

$$
\text { extrema }(x)= \begin{cases}x_{\max }, & \text { for positive logistic map } \\ x_{\min }, & \text { for negative logistic map. }\end{cases}
$$

The design problem involves constraints which are the values of $\operatorname{par}_{b 1}$ and par $_{\text {max }}$, in addition to $\operatorname{par}_{b 2}$ if exist, that is, in the case of positive or negative logistic maps. For single sign maps, par $_{\max }=4 \operatorname{par}_{b 1}$ and $\operatorname{par}_{b 2}=3 \mathrm{par}_{b 1}$, while for alternating sign maps $\operatorname{par}_{\max }=2 \mathrm{par}_{b 1}$. In addition, the values of $x_{\min }$ and $x_{\max }$, in the case of alternating sign maps, are related. For the bifurcation diagram with respect to $\lambda, x_{\max }-$ $x_{\min }=2 a / b$, while for the bifurcation diagram with respect to $a, x_{\max }-x_{\min }=4 /(\lambda b)$. For mostly positive map, $x_{\max }=$ $-3 x_{\min }$, while for mostly negative map, $x_{\max }=-(1 / 3) x_{\min }$. Table 3 summarizes these constraints.

Four different cases of designing a map with certain specifications on the bifurcation diagram versus either parameter are provided in Table 4(a). The design examples have been chosen such that they cover the cases of horizontal and independent scaling, since vertical scaling and zooming maps have been discussed thoroughly. First, the suitable map is chosen according to the required range of output: positive only, negative only, or alternating sign. Then, the design equations are solved simultaneously to get the corresponding values of the other parameters. The bifurcation diagrams, their main key points, and the map equation are shown in Table 4(b).

One of the applications of chaotic maps is generation of sequences of random numbers which are useful in many applications. These are called pseudorandom sequences; that is, although the output appears to be random, it is actually generated deterministically based on a seed value. Seeds are important when reproducible sequences are required, for example, debugging a simulation or encrypting/decrypting a message. Mixing the message with the chaotic sequence achieves desirable cryptographic properties of diffusion and confusion due to the high sensitivity of this sequence to changes in initial conditions. The output pattern after mixing 

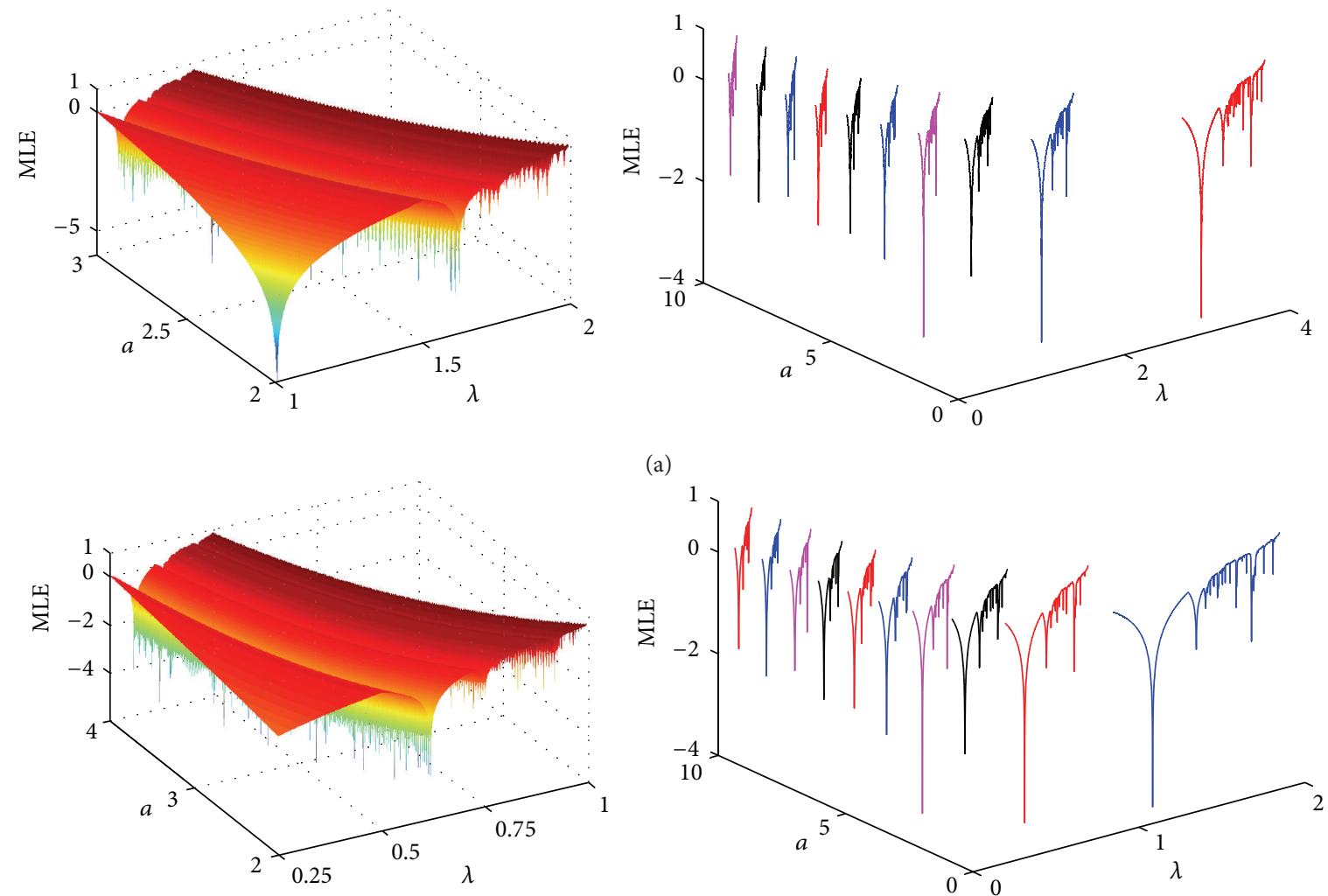

(b)

FIGURE 15: MLE as a function of both $\lambda$ and $a$ for zooming (a) positive and (b) mostly positive maps.

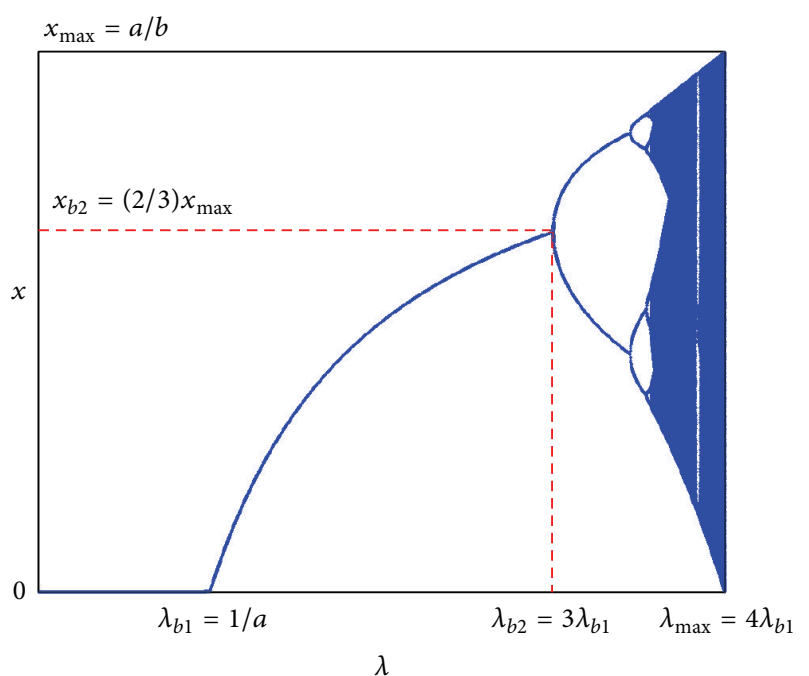

(a)

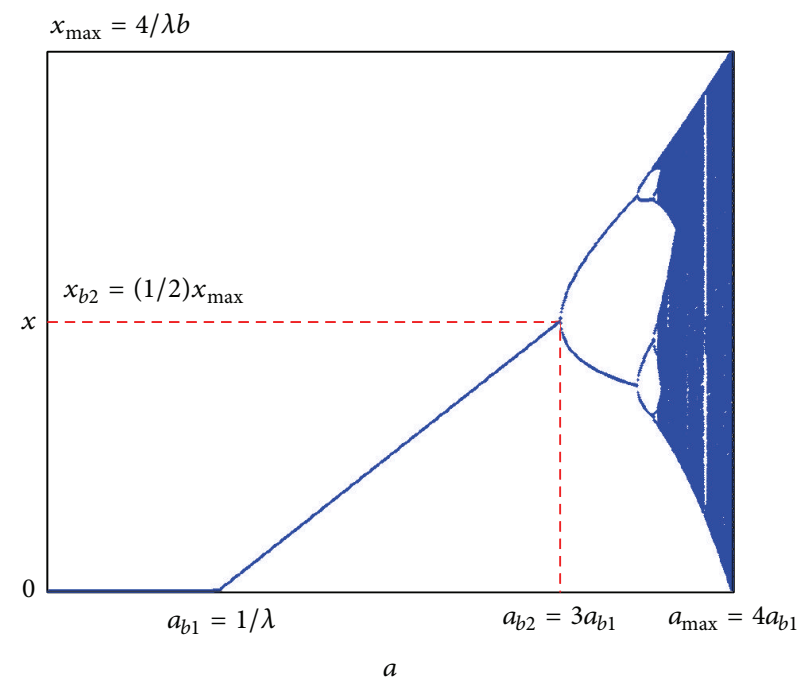

(b)

FIGURE 16: General bifurcation diagrams of independent scaling positive logistic map (a) versus $\lambda$ and (b) versus $a$.

TABLE 3: Constraints of the design problem.

\begin{tabular}{|c|c|c|c|}
\hline & & Single sign maps & Alternating sign maps \\
\hline Constraints on parameter & & $\begin{array}{c}\operatorname{par}_{\text {max }}=4 \operatorname{par}_{b 1} \\
\operatorname{par}_{b 2}=3 \operatorname{par}_{b 1}\end{array}$ & $\begin{aligned} \operatorname{par}_{\max } & =2 \operatorname{par}_{b 1} \\
& -\end{aligned}$ \\
\hline Constraints on output & $\begin{array}{l}\lambda \text {-bifurcation } \\
a \text {-bifurcation }\end{array}$ & $\begin{aligned} \mid \text { extrema }(x) \mid & =\frac{a}{b} \\
|\operatorname{extrema}(x)| & =\frac{4}{(\lambda b)}\end{aligned}$ & $\begin{array}{c}x_{\max }-x_{\min }=\frac{2 a}{b} \\
x_{\max }-x_{\min }=\frac{4}{(\lambda b)}\end{array}$ \\
\hline
\end{tabular}


TABLE 4: Four design cases of the logistic map.

(a)

\begin{tabular}{|c|c|c|c|c|}
\hline Example & Specifications & Suitable map & $\lambda$-bifurcation design & $a$-bifurcation design \\
\hline (a) & $\begin{array}{l}x \geq 0 \\
x_{\max }=1 \\
\operatorname{par}_{b 1}=0.2\end{array}$ & Positive & $a=5, b=5$ & $\lambda=5, b=\frac{4}{5}$ \\
\hline (b) & $\begin{array}{l}x \leq 0 \\
x_{\text {min }}=-\frac{3}{2} \\
\text { par }_{\text {max }}=\frac{4}{3}\end{array}$ & Negative & $a=3, b=2$ & $\lambda=3, b=\frac{8}{9}$ \\
\hline (c) & $\begin{array}{l}x_{\min }=-0.25 \\
\left|\frac{x_{\max }}{x_{\min }}\right|>1 \\
\text { par }_{\max }=1\end{array}$ & Mostly positive & $a=2, b=4$ & $\lambda=2, b=2$ \\
\hline (d) & $\begin{array}{l}x_{\max }=1 \\
\left|\frac{x_{\max }}{x_{\min }}\right|<1 \\
\operatorname{par}_{b 1}=1.5\end{array}$ & Mostly negative & $a=\frac{2}{3}, b=\frac{1}{3}$ & $\lambda=\frac{2}{3}, b=\frac{3}{2}$ \\
\hline
\end{tabular}

(b)

Bifurcation diagram with respect to $\lambda$

$$
f(x)=\lambda x(5-5 x)
$$

(a)

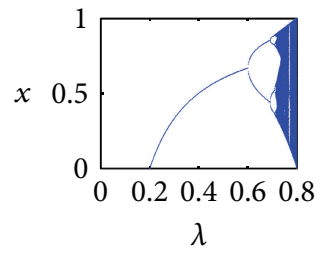

$$
\begin{aligned}
\lambda_{b 1} & =0.2 \\
\lambda_{b 2} & =0.6 \\
\lambda_{\max } & =0.8 \\
x_{b 2} & =\frac{2}{3} \\
x_{\max } & =1
\end{aligned}
$$

Bifurcation diagram with respect to $a$

Horizontal scaling

$$
\begin{gathered}
f(x)=\lambda x(3+2 x) \\
\lambda_{b 1}=\frac{1}{3} \\
\lambda_{b 2}=1 \\
\lambda_{\text {max }}=\frac{4}{3} \\
x_{b 2}=-1 \\
x_{\text {min }}=-1.5
\end{gathered}
$$

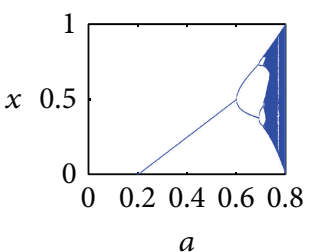

$$
\begin{gathered}
f(x)=5 x\left(a-\frac{4}{5} x\right) \\
a_{b 1}=0.2 \\
a_{b 2}=0.6 \\
a_{\max }=0.8 \\
x_{b 2}=0.5 \\
x_{\max }=1
\end{gathered}
$$

Horizontal scaling

$$
\begin{gathered}
f(x)=3 x\left(a+\frac{8}{9} x\right) \\
a_{b 1}=\frac{1}{3} \\
a_{b 2}=1 \\
a_{\max }=\frac{4}{3} \\
x_{b 2}=-0.75 \\
x_{\min }=-1.5
\end{gathered}
$$

Independent scaling

$$
\begin{gathered}
f(x)=-\lambda x(2-4 x) \\
\lambda_{b 1}=0.5 \\
\lambda_{\text {max }}=1 \\
x_{\text {min }}=-0.25 \\
x_{\text {max }}=0.75
\end{gathered}
$$

Independent scaling

$$
\begin{gathered}
f(x)=-\lambda x\left(\frac{2}{3}+\frac{1}{3} x\right) \\
\lambda_{b 1}=1.5 \\
\lambda_{\max }=3 \\
x_{\min }=-3 \\
x_{\max }=1
\end{gathered}
$$

Independent scaling

$f(x)=-2 x(a-2 x)$

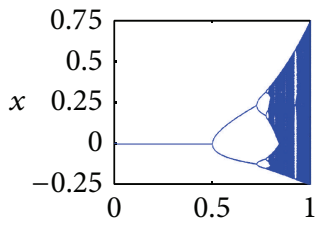

$a_{b 1}=0.5$

$a_{\max }=1$

$x_{\text {min }}=-0.25$

$x_{\max }=0.75$

Independent scaling

$$
\begin{gathered}
f(x)=-\frac{2}{3} x\left(a+\frac{3}{2} x\right) \\
a_{b 1}=1.5 \\
a_{\max }=3 \\
x_{\min }=-3 \\
x_{\max }=1
\end{gathered}
$$

Independent scaling 


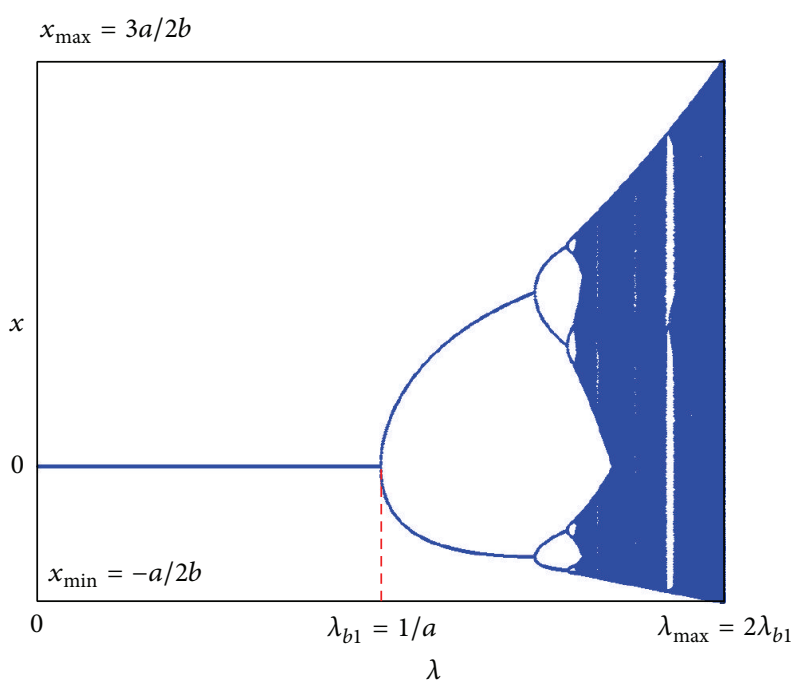

(a)

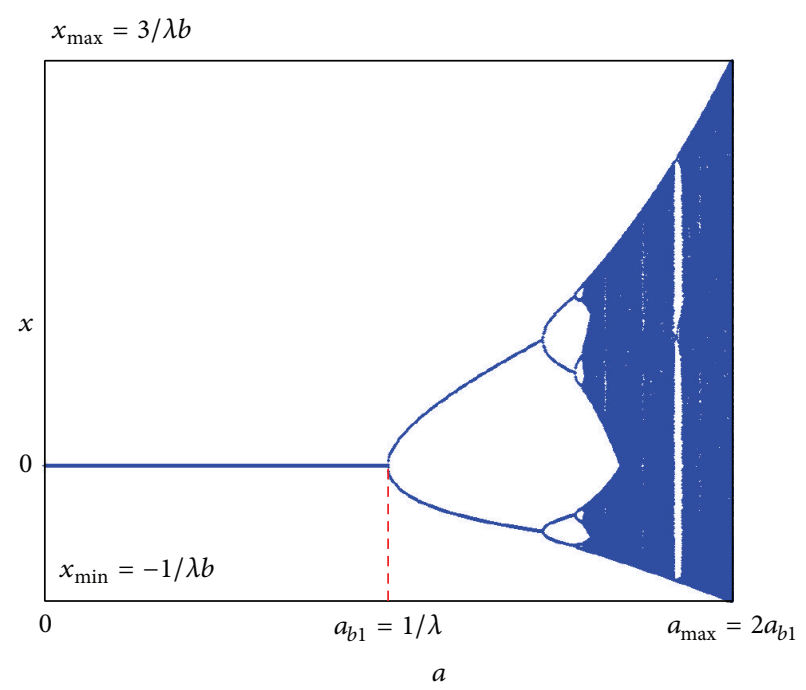

(b)

FIGURE 17: General bifurcation diagrams of independent scaling mostly positive logistic map (a) versus $\lambda$ and (b) versus $a$.

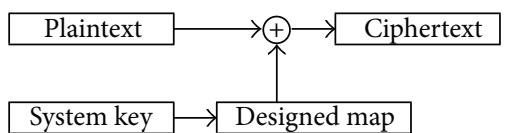

(a)

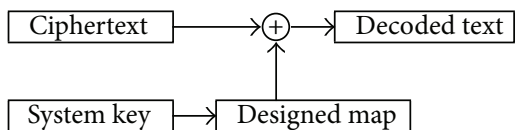

(b)

FIGURE 18: Simple text encryption system (a) encryption scheme and (b) decryption scheme.

TABLE 5: System key used in the encryption scheme.

\begin{tabular}{lccc}
\hline$x_{\text {key }}$ & $a_{\text {key }}$ & $b_{\text {key }}$ & $\lambda_{\text {key }}$ \\
\hline 32 bits & 32 bits & 32 bits & 32 bits \\
\hline
\end{tabular}

is called the ciphertext which is an encrypted combination of the key and the plaintext, where the key is a secret and the plaintext is the message. Moreover, successive iterations of a chaotic system reduce the statistical dependency of the ciphertext on the plaintext. Such applications require certain specifications on the bifurcation diagram in terms of the parameter range, in addition to the output range. The encryption system used to validate our designs is explained as follows.

5.2. Encryption System. The encryption scheme used is based on simple xor operation between the output sequence of the map and the characters constituting the plain text as shown in Figure 18. Moreover, the extra parameters $a$ and $b$ in addition to the system parameter $\lambda$ and the initial condition $x_{0}$ can be used to construct a more efficient encryption key. The key consists of four parameters each represented in 32 bits, that is, a total of 128 bits as shown in Table 5 . The calculation of each parameter $S$ and initial value $x_{0}$ are given by

$$
\begin{aligned}
S & =S_{\mathrm{fix}}-S_{\mathrm{key}} \times 10^{-12}, \\
x_{0} & = \pm x_{\mathrm{key}} \times 10^{-10}
\end{aligned}
$$

where $S_{\text {fix }}$ is the fixed part of this parameter, $S_{\text {key }}$ is the decimal value of the corresponding 32 bits shown in Table 5 , and $x_{\text {key }}$ is the decimal value given in the key. The scaling factor $10^{-12} \simeq 2^{-40}$ is used after converting to double precision floating-point to ensure that the value of $S_{\text {key }}$ does not affect the digits of $S_{\text {fix }}$. Similarly, a scaling factor of $10^{-10} \simeq 2^{-34}$ is used for calculating $x_{0}$ from $x_{\mathrm{key}}$. The sign of $x_{0}$ is chosen to limit the initial values within the allowable range according to the map characteristics (single or alternating sign map). In order to enhance the characteristics of the system, the double precision floating-point representation of each map output is subdivided into eight blocks which are xored at first and then the output is xored with a character from the plain text and so on till the last character. Table 6 shows the encrypted HEX code corresponding to each designed map; that is, the fixed values of the three parameters are set to the values given in Table 4 with $\lambda_{\max }$ or $a_{\max }$ corresponding to maximum chaotic behavior. We use the famous quote by Ghandi: "You must be the change you wish to see in the world." as the plaintext. The encryption key is chosen as "B93E61A2A2F49CB58EA37B51C49A5E68". Encryption schemes that offer better performance utilizing such simple maps usually use innovative combinations of them. Such combinations could be even suitable for more complicated image encryption applications, for example, the encryption techniques presented in $[36,61]$. 
TABle 6: Encrypted HEX code for each design.

\begin{tabular}{ll}
\hline Plaintext & You must be the change you wish to see in the world. \\
\hline HEX code & 596F75206D75737420626520746865206368616E676520796F75 \\
& 207769736820746F2073656520696E2074686520776F726C642E \\
\hline Encrypted & \\
HEX code & \\
Design $(a)$ with respect to $\lambda$ & 8F3B4F398DF8F5E61E20E1EC2879EFF53C1F0F04AF90FEB6292F \\
$\left(\lambda_{\text {fix }}, a_{\text {fix }}, b_{\text {fix }}\right)=\left(\frac{4}{a}, 5,5\right)$ & 06CBA9A1C2B13C942A84D28CDA033A15373587775A5675008032 \\
\hline Design $(a)$ with respect to $a$ & 8FBF3C41C6BD1FD8D4C269F8A0D731CA8DB0AD0E79D6D7FE5140 \\
$\left(\lambda_{\text {fix }}, a_{\text {fix }}, b_{\text {fix }}\right)=\left(5, \frac{4}{\lambda}, 0.8\right)$ & 9E9B62B5A32F8747504B940DF967B99E45BFAE0EA1576A6EC3EB \\
\hline Design $(b)$ with respect to $\lambda$ & 0F0B2C34DCEE02A639B528B5D3D78305A3C0F4B6A7E5F0B1157E \\
$\left(\lambda_{\text {fix }}, a_{\text {fix }}, b_{\text {fix }}\right)=\left(\frac{4}{a}, 3,2\right)$ & 8134C06EF6E6C1250BA656916741DB0011D86C69E5F1C1A564F6 \\
\hline Design $(b)$ with respect to $a$ & 0FEC095719D3625223CD7941B2571AFEBE7E2B649BE901F76D81 \\
$\left(\lambda_{\text {fix }}, a_{\text {fix }}, b_{\text {fix }}\right)=\left(3, \frac{4}{\lambda}, \frac{8}{9}\right)$ & DC77ADBEE5450129D9FF0D0B71B963F64C2FEED57AEDF3DCC2A3 \\
\hline Design $(c)$ with respect to $\lambda$ & 8FEC4A6CBDFC371B4183EA5FBE09B81217CAEAFF015332F7CB87 \\
$\left(\lambda_{\text {fix }}, a_{\text {fix }}, b_{\text {fix }}\right)=\left(\frac{2}{a}, 2,4\right)$ & E34E3B7F1F9287AEC651E015876E9BAD40BBC222BEC2A63803C3 \\
\hline Design $(c)$ with respect to $a$ & 8F919CFC52131AA8E987F7D4DC75133A1E81DD58317886F57EBB \\
$\left(\lambda_{\text {fix }}, a_{\text {fix }}, b_{\text {fix }}\right)=\left(2, \frac{2}{\lambda}, 2\right)$ & 5553A8AD90B643E204628793FC095E895248EF8452E8CFD8D1D4 \\
\hline Design $(d)$ with respect to $\lambda$ & 0F9A0D7FB4449B8F590E88D8248AF929CEC7EDAA7C1274AB83D1 \\
$\left(\lambda_{\text {fix }}, a_{\text {fix }}, b_{\text {fix }}\right)=\left(\frac{2}{a}, \frac{2}{3}, \frac{1}{3}\right)$ & C4A36A5C24B3A8B938984DE2B6D9B26251FF0F1B6D26A8FD13FE \\
\hline Design (d) with respect to $a$ & 0F710918D640BD50302A1ADE60BC4F0BFAC536C2811122A31BB6 \\
$\left(\lambda_{\text {fix }}, a_{\text {fix }}, b_{\text {fix }}\right)=\left(\frac{2}{3}, \frac{2}{\lambda}, \frac{3}{2}\right)$ & B6A37A7C1DB5ADCFA7F31BAE0FA9F1B9F93F2CB127B9862841CF \\
\hline
\end{tabular}

\section{Conclusion}

In this paper, four possible variations on the relation representing the logistic map were proposed. The four maps are called positive logistic map, mostly positive logistic map, negative logistic map, and mostly negative logistic map according to the maximum chaotic range of the output. These variations differ in their characteristics and do not confine the output to a restricted range of fractions between 0 and 1 , thus allowing more flexibility that fits the most recent applications such as quantitative financial modeling, traffic, weather forecasting, and others. Moreover, a general design procedure was proposed for designing logistic maps through a generalized form of the equation that holds two extra parameters. The parameters $(a, b)$ may take one of three cases: $(a, b), a, b \in R^{+}$called the independent scaling case, $(1, b)$ called the vertical scaling case, and $(a, 1)$ called the zooming case. The proposed maps were analyzed from the viewpoint of iteration effect, ranges of $\lambda$ and $x$, the fixed points, the bifurcation diagrams, and the maximum Lyapunov exponent with respect to all system parameters. The general schematic of the bifurcation diagrams with respect to $\lambda$ in addition to a new bifurcation diagram with respect to $a$ were provided. Four different design examples were presented to validate the provided design procedure in addition to testing their efficiency in a simple text encryption application.

\section{Conflict of Interests}

The authors declare that there is no conflict of interests regarding the publication of this paper.

\section{References}

[1] K. M. Cuomo and A. V. Oppenheim, "Circuit implementation of synchronized chaos with applications to communications," Physical Review Letters, vol. 71, no. 1, pp. 65-68, 1993.

[2] A. G. Radwan, A. M. Soliman, and A.-L. El-Sedeek, "An inductorless CMOS realization of Chua's circuit," Chaos, Solitons \& Fractals, vol. 18, no. 1, pp. 149-158, 2003.

[3] A. G. Radwan, A. M. Soliman, and A. El-Sedeek, "MOS realization of the modified Lorenz chaotic system," Chaos, Solitons \& Fractals, vol. 21, no. 3, pp. 553-561, 2004.

[4] S. Mandal and S. Banerjee, "Analysis and CMOS implementation of a chaos-based communication system," IEEE Transactions on Circuits and Systems I: Regular Papers, vol. 51, no. 9, pp. 1708-1722, 2004.

[5] A. G. Radwan, A. M. Soliman, and A. S. Elwakil, "1-D digitallycontrolled multiscroll chaos generator," International Journal of Bifurcation and Chaos, vol. 17, no. 1, pp. 227-242, 2007.

[6] A. S. Mansingka, A. G. Radwan, and K. N. Salama, "Design, implementation and analysis of fully digital 1-D controllable multiscroll chaos," in Proceedings of the 23rd International Conference on Microelectronics (ICM '11), pp. 1-5, IEEE, December 2011. 
[7] M. L. Barakat, A. G. Radwan, and K. N. Salama, "Hardware realization of chaos based block cipher for image encryption," in 2011 23rd International Conference on Microelectronics, ICM 2011, tun, December 2011.

[8] M. A. Zidan, A. G. Radwan, and K. N. Salama, "Random number generation based on digital differential chaos," in Proceedings of the 54th IEEE International Midwest Symposium on Circuits and Systems (MWSCAS '11), pp. 1-4, IEEE, August 2011.

[9] A. S. Mansingka, A. G. Radwan, and K. N. Salama, "Fully digital 1-D, 2-D and 3-D multiscroll chaos as hardware pseudo random number generators," in Proceedings of the IEEE 55th International Midwest Symposium on Circuits and Systems (MWSCAS '12), pp. 1180-1183, IEEE, August 2012.

[10] M. A. Zidan, A. G. Radwan, and K. N. Salama, "Controllable Vshape multiscroll butterfly attractor: system and circuit implementation," International Journal of Bifurcation and Chaos, vol. 22, no. 6, Article ID 1250143, 13 pages, 2012.

[11] R. M. May, "Simple mathematical models with very complicated dynamics," Nature, vol. 261, no. 5560, pp. 459-467, 1976.

[12] P. F. Verhulst, "Notice sur la loi que la population suit dans son accroissement," Correspondance Mathématique et Physique de l'Observatoire de Bruxelles Publiée Part A: Quetelet, vol. 10, pp. $113-121,1838$.

[13] M. J. Feigenbaum, "Quantitative universality for a class of nonlinear transformations," Journal of Statistical Physics, vol. 19, no. 1, pp. 25-52, 1978.

[14] A. L. Lloyd, "The coupled logistic map: a simple model for the effects of spatial heterogeneity on population dynamics," Journal of Theoretical Biology, vol. 173, no. 3, pp. 217-230, 1995.

[15] S. H. Strogatz, Nonlinear Dynamics And Chaos: With Applications to Physics, Biology, Chemistry, and Engineering, Westview Press, 2014.

[16] E. Scholl, Nonlinear Spatio-Temporal Dynamics and Chaos in Semiconductors, vol. 10, Cambridge University Press, 2001.

[17] N. K. Pareek, V. Patidar, and K. K. Sud, "Image encryption using chaotic logistic map," Image and Vision Computing, vol. 24, no. 9, pp. 926-934, 2006.

[18] W. Yao, P. Ye, and X. Li, "An effective privacy-preserving algorithm based on logistic map and rubik's cube transformation," Discrete Dynamics in Nature and Society, vol. 2014, Article ID 178585, 11 pages, 2014.

[19] S. C. Phatak and S. S. Rao, "Logistic map: a possible randomnumber generator," Physical Review E, vol. 51, no. 4, 1995.

[20] L. Kocarev and G. Jakimoski, "Logistic map as a block encryption algorithm," Physics Letters. A, vol. 289, no. 4-5, pp. 199-206, 2001.

[21] A. Kanso and N. Smaoui, "Logistic chaotic maps for binary numbers generations," Chaos, Solitons \& Fractals, vol. 40, no. 5, pp. 2557-2568, 2009.

[22] V. Patidar, K. K. Sud, and N. K. Pareek, "A pseudo random bit generator based on chaotic logistic map and its statistical testing," Informatica, vol. 33, no. 4, pp. 441-452, 2009.

[23] N. Singh and A. Sinha, "Chaos-based secure communication system using logistic map," Optics and Lasers in Engineering, vol. 48, no. 3, pp. 398-404, 2010.

[24] M. Suneel, "Electronic circuit realization of the logistic map," Sadhana, vol. 31, no. 1, pp. 69-78, 2006.

[25] J. Gu and S. Chen, "Nonlinear analysis on traffic flow based on catastrophe and chaos theory," Discrete Dynamics in Nature and Society, vol. 2014, Article ID 535167, 11 pages, 2014.
[26] D. A. Hsieh, "Chaos and nonlinear dynamics: application to financial markets," The Journal of Finance, vol. 46, no. 5, pp. 1839-1877, 1991.

[27] N. Basalto, R. Bellotti, F. De Carlo, P. Facchi, and S. Pascazio, "Clustering stock market companies via chaotic map synchronization," Physica A: Statistical Mechanics and Its Applications, vol. 345, no. 1-2, pp. 196-206, 2005.

[28] T. Chen, J. He, and Q. Yin, "Dynamics evolution of credit risk contagion in the CRT market," Discrete Dynamics in Nature and Society, vol. 2013, Article ID 206201, 9 pages, 2013.

[29] K. T. Alligood, T. D. Sauer, and J. A. Yorke, Chaos: An Introduction to Dynamical Systems, Springer, 1996.

[30] R. Matthews, "On the derivation of a 'chaotic' encryption algorithm," Cryptologia, vol. 13, no. 1, pp. 29-42, 1989.

[31] Z. Elhadj and J. C. Sprott, "The effect of modulating a parameter in the logistic map," Chaos, vol. 18, no. 2, Article ID 023119, 2008.

[32] R. Vázquez-Medina, A. Díaz-Méndez, J. L. del Río-Correa, and J. López-Hernández, "Design of chaotic analog noise generators with logistic map and MOS QT circuits," Chaos, Solitons \& Fractals, vol. 40, no. 4, pp. 1779-1793, 2009.

[33] E. A. Levinsohn, S. A. Mendoza, and E. Peacock-López, "Switching induced complex dynamics in an extended logistic map," Chaos, Solitons \& Fractals, vol. 45, no. 4, pp. 426-432, 2012.

[34] A. G. Radwan, "On some generalized discrete logistic maps," Journal of Advanced Research, vol. 4, no. 2, pp. 163-171, 2013.

[35] A. G. Radwan and S. K. Abd-El-Hafiz, "Image encryption using generalized tent map," in Proceedings of the IEEE 20th International Conference on Electronics, Circuits, and Systems (ICECS '13), pp. 653-656, IEEE, December 2013.

[36] A. G. Radwan, S. K. Abd-El-Hafiz, and S. H. AbdElHaleem, "An image encryption system based on generalized discrete maps," in Proceedings of the 21st IEEE International Conference on Electronics, Circuits and Systems (ICECS '14), pp. 283-286, IEEE, Marseille, France, December 2014.

[37] D. Orrell, L. Smith, J. Barkmeijer, and T. N. Palmer, "Model error in weather forecasting," Nonlinear Processes in Geophysics, vol. 8, no. 6, pp. 357-371, 2001.

[38] A. Trevisan and L. Palatella, "Chaos and weather forecasting: the role of the unstable subspace in predictability and state estimation problems," International Journal of Bifurcation and Chaos, vol. 21, no. 12, pp. 3389-3415, 2011.

[39] D. Dangoisse, P. Glorieux, and D. Hennequin, "Chaos in a $\mathrm{CO}_{2}$ laser with modulated parameters: experiments and numerical simulations," Physical Review A, vol. 36, no. 10, article 4775, 1987.

[40] M. D. Bernardo and F. Vasca, "Discrete-time maps for the analysis of bifurcations and chaos in DC/DC converters," IEEE Transactions on Circuits and Systems I: Fundamental Theory and Applications, vol. 47, no. 2, pp. 130-143, 2000.

[41] A. N. Kolmogorov, Foundations of the Theory of Probability, Chelsea Publishing Company, New York, NY, USA, 1950.

[42] A. Khrennikov, Interpretations of Probability, Walter De Gruyter, 2009.

[43] Y. D. Han, W. Y. Hwang, and I. G. Koh, "Explicit solutions for negative-probability measures for all entangled states," Physics Letters A, vol. 221, no. 5, pp. 283-286, 1996.

[44] T. Curtright and C. Zachos, "Negative probability and uncertainty relations," Modern Physics Letters A: Particles and Fields, Gravitation, Cosmology, Nuclear Physics, vol. 16, no. 37, pp. 2381$2385,2001$. 
[45] H. F. Hofmann, "How to simulate a universal quantum computer using negative probabilities," Journal of Physics. A. Mathematical and Theoretical, vol. 42, no. 27, Article ID 275304, pp. 275-304, 2009.

[46] M. O. Scully, H. Walther, and W. Schleich, "Feynman's approach to negative probability in quantum mechanics," Physical Review A, vol. 49, no. 3, pp. 1562-1566, 1994.

[47] E. Wigner, "On the quantum correction for thermodynamic equilibrium," Physical Review, vol. 40, no. 5, pp. 749-759, 1932.

[48] P. Dirac, "Bakerian lecture. The physical interpretation of quantum mechanics," Proceedings of the Royal Society of London. Series A, vol. 180, no. 980, pp. 1-40, 1942.

[49] R. P. Feynman, "Negative probability," in Quantum Implications: Essays in Honour of David Bohm, F. D. Peat and B. Hiley, Eds., pp. 235-248, Routledge \& Kegan Paul, 1987.

[50] W. Mückenheim, "A review of extended probabilities," Physics Reports: A Review Section of Physics Letters, vol. 133, no. 6, pp. 337-401, 1986.

[51] T. Rothman and E. C. Sudarshan, "Hidden variables or positive probabilities?" International Journal of Theoretical Physics, vol. 40, no. 8, pp. 1525-1543, 2001.

[52] C. Ferrie and J. Emerson, "Frame representations of quantum mechanics and the necessity of negativity in quasi-probability representations," Journal of Physics A: Mathematical and Theoretical, vol. 41, no. 35, Article ID 352001, 11 pages, 2008.

[53] M. Gell-Mann and J. B. Hartle, "Decoherent histories quantum mechanics with one real fine-grained history," Physical Review A: Atomic, Molecular, and Optical Physics, vol. 85, no. 6, Article ID 062120, 2012.

[54] P. Dirac, "Quantum electrodynamics," Communications of the Dublin Institute for Advanced Studies Series A, vol. 1, pp. 1-36, 1943.

[55] R. A. Jarrow and S. M. Turnbull, "Pricing derivatives on financial securities subject to credit risk," The Journal of Finance, vol. 50, no. 1, pp. 53-85, 1995.

[56] D. Duffie and K. J. Singleton, "Modeling term structures of defaultable bonds," Review of Financial Studies, vol. 12, no. 4, pp. 687-720, 1999.

[57] E. G. Haug, "Why so negative to negative probabilities?" Wilmott Magazine, pp. 34-38, 2004.

[58] J. C. Cox, S. A. Ross, and M. Rubinstein, "Option pricing: a simplified approach," Journal of Financial Economics, vol. 7, no. 3, pp. 229-263, 1979.

[59] M. Burgin and G. Meissner, "Negative probabilities in financial modeling," Wilmott, vol. 2012, no. 58, pp. 60-65, 2012.

[60] J. T. Ambadan and K. B. Joseph, "Asymmetrical mirror bifurcations in logistic map with a discontinuity at zero," in Proceedings of the National Conference on Nonlinear Systems and Dynamics (NCNSD '06), February 2006.

[61] Z.-L. Zhu, W. Zhang, K.-W. Wong, and H. Yu, "A chaosbased symmetric image encryption scheme using a bit-level permutation," Information Sciences, vol. 181, no. 6, pp. 1171-1186, 2011. 


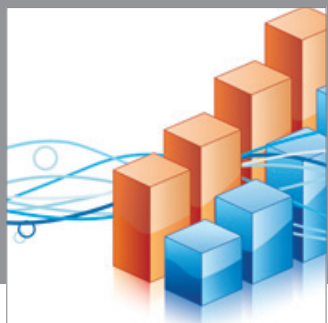

Advances in

Operations Research

mansans

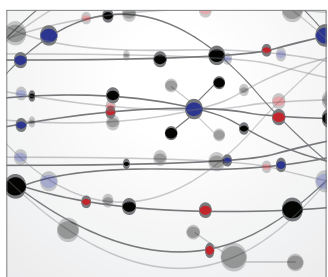

The Scientific World Journal
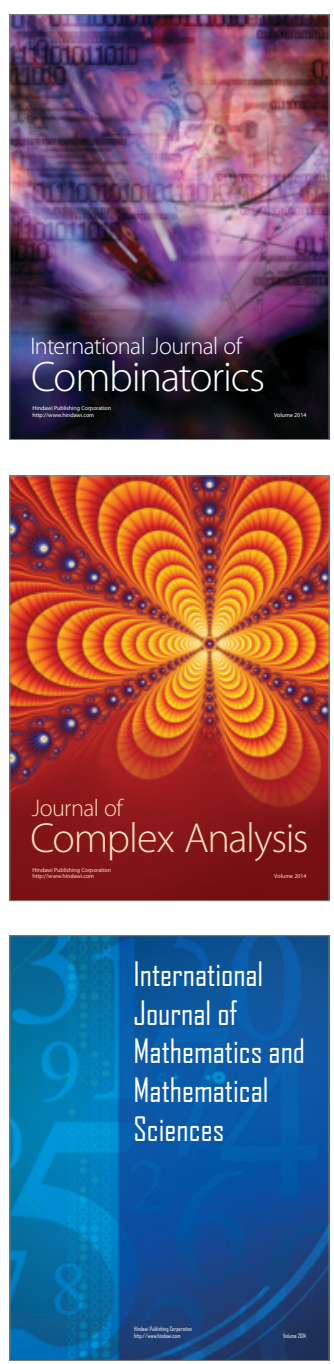
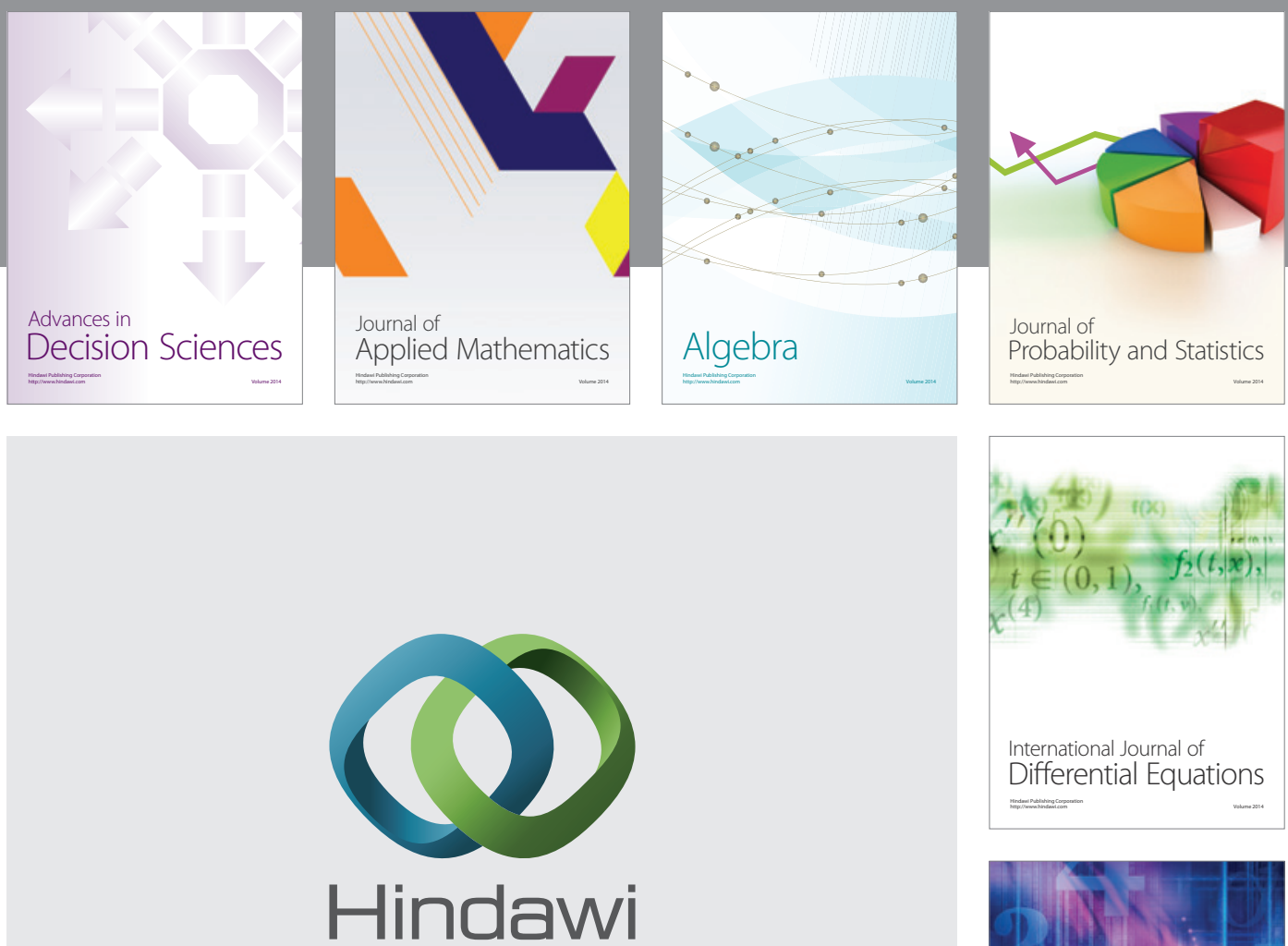

Submit your manuscripts at http://www.hindawi.com
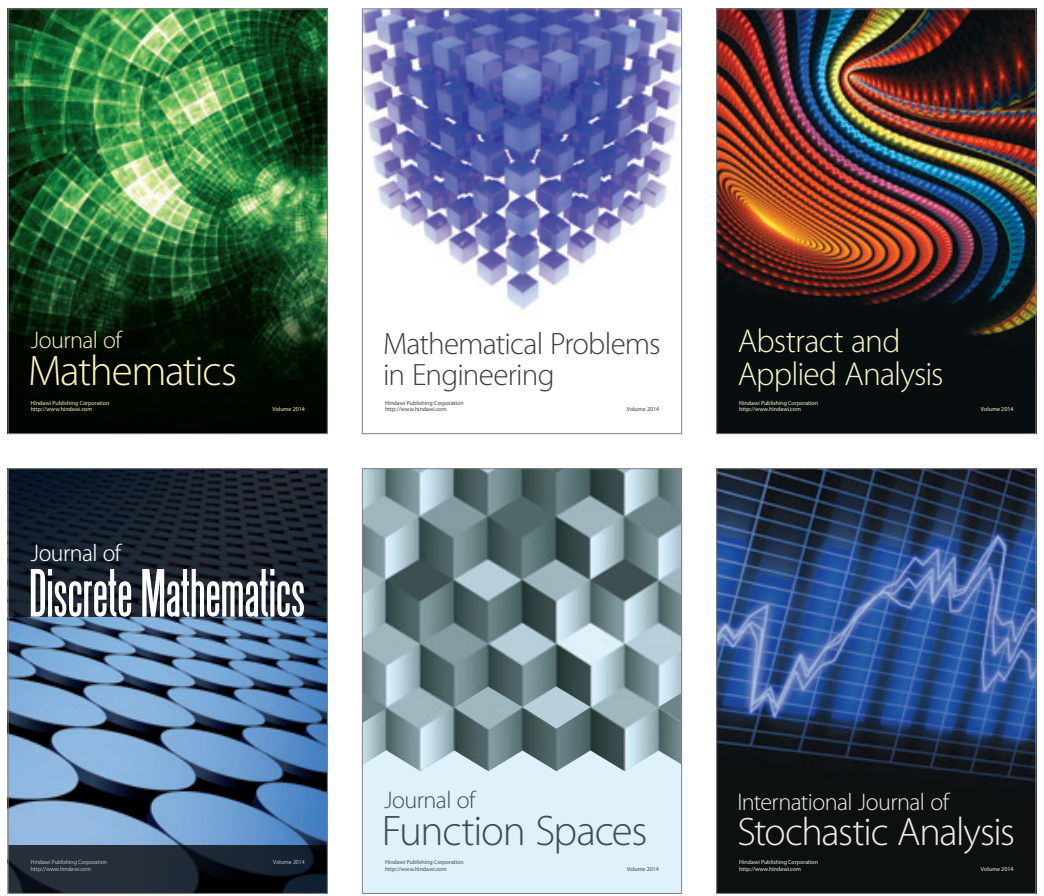

Journal of

Function Spaces

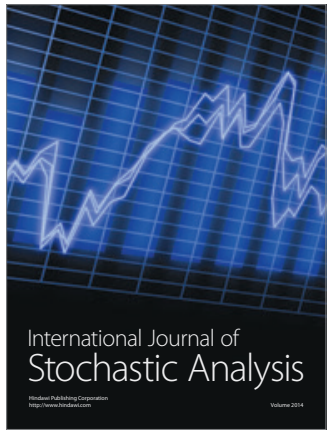

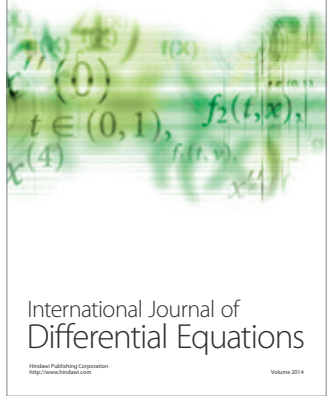
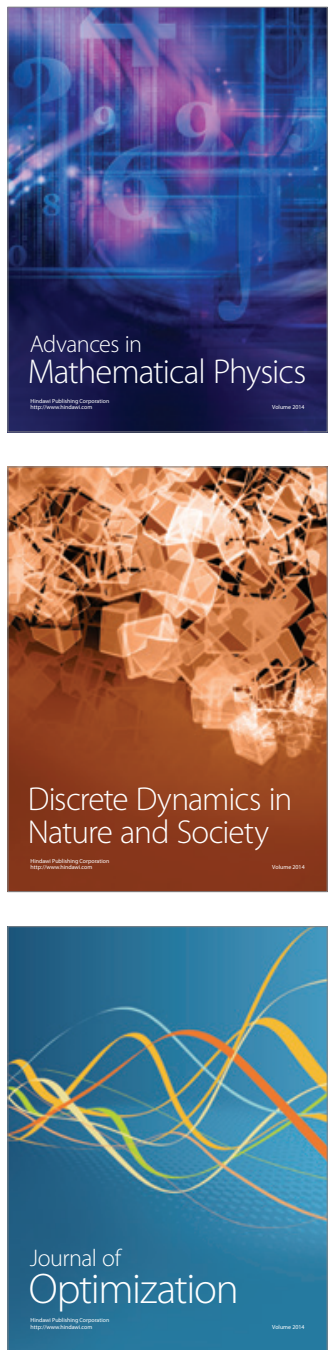